\title{
Hessian measures of semi-convex functions and applications to support measures of convex bodies*
}

\author{
Andrea Colesanti and Daniel Hug
}

\begin{abstract}
This paper originates from the investigation of support measures of convex bodies (sets of positive reach), which form a central subject in convex geometry and also represent an important tool in related fields. We show that these measures are absolutely continuous with respect to Hausdorff measures of appropriate dimensions, and we determine the Radon-Nikodym derivatives explicitly on sets of $\sigma$-finite Hausdorff measure. The results which we obtain in the setting of the theory of convex bodies (sets of positive reach) are achieved as applications of various new results on Hessian measures of convex (semi-convex) functions. Among these are a Crofton formula, results on the absolute continuity of Hessian measures, and a duality theorem which relates the Hessian measures of a convex function to those of the conjugate function. In particular, it turns out that curvature and surface area measures of a convex body $K$ are the Hessian measures of special functions, namely the distance function and the support function of $K$.
\end{abstract}

\section{Introduction}

The theory of curvature and surface area measures plays a central rôle in the classical theory of convex bodies. The importance of these measures is due to the fact that they relate classical convexity to integral and stochastic geometry, for which they are indispensible tools. But they are also relevant for such diverse subjects as the theory of valuations on convex bodies [38], [3], [4], research on zonoids, projection functions and functional analytic characterizations of classes of convex bodies [26], [27], geometric tomography [22], or the study of isotropic positions of convex bodies [23], [24], and thus the local theory of Banach spaces. Despite the fact that these measures have a long tradition in convexity, some of the basic questions only have been addressed quite recently and others have not been solved so far; this can be seen, for instance, from the survey papers [39], [37] and the lecture notes [40].

*AMS 1991 subject classification. Primary 52A20, 26B25; Secondary 53C65, 28A78.

Key words and phrases. Hessian measure, support measure, curvature and surface area measure, Crofton formula, absolute continuity, Radon-Nikodym derivative, semi-convex function, set of positive reach, Hausdorff measure. 
The curvature and surface area measures, and also their common generalization, the support measures, contain essential local as well as global information about the shape of the set with which they are associated. The investigation of the connection between measure theoretic properties of these measures and geometric properties of the underlying sets represents a major theme in this field; see [40] and the survey given in Section 1 of [29]. Another main topic is the study of integral geometric formulae such as principal kinematic formulae or Crofton formulae and their applications to stochastic geometry. Of course, curvature and surface area measures also lead to interesting and challenging questions from a mainly measure theoretic point of view; in particular, various relationships between these measures and Hausdorff measures have been analyzed.

The present paper originates from the investigation of properties of curvature and surface area measures of convex bodies. The main purpose is to establish absolute continuity results of such measures with respect to Hausdorff measures of suitable dimensions, and to find explicit expressions for the corresponding densities. The results that we obtain in the setting of the theory of convex bodies are achieved as applications of new and more general results, presented in the first part of the paper, regarding Hessian measures of semi-convex functions. Hessian measures have only emerged quite recently, and most likely they have the potential for a similar development as the classical curvature and surface area measures. It is a major aim of this investigation to improve our knowledge of these measures, which is particularly rewarding, since it also leads to new insights into the existing theory of curvature and surface area measures.

If $K \subset \mathbb{R}^{d}$ is a convex body, i.e. a non-empty compact convex set, then its curvature measures $C_{j}(K, \cdot), j \in\{0,1, \ldots, d-1\}$, are Borel measures on $\mathbb{R}^{d}$ which are concentrated on the boundary $\partial K$ of $K$ and which generalize the notion of surface integrals of the elementary symmetric functions of the principal curvatures of $\partial K$. An analogous notion is the one of a surface area measure of $K$, denoted by $S_{j}(K, \cdot)$, where $j \in\{0,1, \ldots, d-1\}$; the surface area measures are defined on the Borel sets of the unit sphere $S^{d-1}$, and for convex bodies with sufficiently smooth support functions they correspond to integrals of the elementary symmetric functions of the principal radii of curvature. The concept of support measures, or generalized curvature measures, represents a unified approach to curvature and surface area measures. We refer the reader to the book [36] for a detailed presentation of these measures (see Chapter 4), for further references to the extensive literature on the subject, as well as for a description of all notions of convex geometry for which no explicit definition is given here.

For special classes of convex bodies, such as polytopes or sufficiently smooth convex bodies, the properties of curvature and surface area measures are clear from the explicit representations of these measures, which can be obtained in these particular cases. However, the situation changes drastically when we consider arbitrary convex bodies. One of the general measure theoretic results, due to R. Schneider (see Theorem 4.6.5 in [36]), states that the measure $C_{j}(K, \cdot)$ is absolutely continuous with respect to the $j$-dimensional Hausdorff measure $\mathcal{H}^{j}$ for every $j \in\{0,1, \ldots, d-1\}$; analogously, $S_{j}(K, \cdot)$ is absolutely continuous with respect to $\mathcal{H}^{d-j-1}$, for every $j \in\{0,1, \ldots, d-1\}$ (see the Notes for Section 4.6 in [36]). As a consequence, $C_{j}(K, \cdot)$ and $S_{j}(K, \cdot)$ possess Radon-Nikodym derivatives 
with respect to $\mathcal{H}^{j}$ and $\mathcal{H}^{d-j-1}$, respectively, on sets of $\sigma$-finite Hausdorff measure.

In this paper we present another proof of the absolute continuity results mentioned above; moreover, our approach will enable us to find the explicit values of the corresponding Radon-Nikodym derivatives, and thus we see how the singularities of the convex bodies involved are related to the values of these measures. Indeed we prove the following theorem which states that the curvature and surface area measures of a convex body $K$, evaluated for sets having $\sigma$-finite Hausdorff measure, can be calculated as weighted Hausdorff measures and the weights measure the strength of the singularities of $K$.

Theorem 0.1 Let $K \subset \mathbb{R}^{d}$ be a convex body and let $j \in\{0,1, \ldots, d-1\}$. If $\alpha \subset \partial K$ is a Borel set having $\sigma$-finite $j$-dimensional Hausdorff measure, then

$$
\left(\begin{array}{c}
d-1 \\
j
\end{array}\right) C_{j}(K, \alpha)=\int_{\alpha} \mathcal{H}^{d-1-j}\left(N(K, x) \cap S^{d-1}\right) d \mathcal{H}^{j}(x)
$$

where $N(K, x)$ stands for the normal cone of $K$ at $x$. Furthermore, let $\omega \subset S^{d-1}$ be a Borel set having $\sigma$-finite $(d-j-1)$-dimensional Hausdorff measure, then

$$
\left(\begin{array}{c}
d-1 \\
j
\end{array}\right) S_{j}(K, \omega)=\int_{\omega} \mathcal{H}^{j}(F(K, u)) d \mathcal{H}^{d-j-1}(x)
$$

where $F(K, u):=\{x \in \partial K: u \in N(K, x)\}$ for $u \in S^{d-1}$.

For a convex body $K \subset \mathbb{R}^{d}$, let us denote by

$$
\Sigma^{j}(K):=\{x \in \partial K: \operatorname{dim}(N(K, x)) \geq d-j\}
$$

the set of $j$-singular boundary points of $K$, and let

$$
\Sigma_{j}(K):=\left\{u \in S^{d-1}: \operatorname{dim}(F(K, u)) \geq d-j-1\right\}
$$

be the set of $j$-singular normal vectors of $K$, for $j \in\{0,1, \ldots, d-1\}$. Then Theorem 0.1 implies that the $j$-th curvature measure of $K$ does not vanish on a set $\alpha$ of $\sigma$-finite $j$-dimensional Hausdorff measure if and only if $\alpha$ contains a set of $j$-singular points having positive $j$-dimensional Hausdorff measure; a corresponding statement is true for surface area measures.

Theorem 0.1 improves and completes Theorem 3.2 and Theorem 4.3 in [28], where the author found the value of the densities of $C_{j}(K, \cdot)$ and $S_{j}(K, \cdot)$ on $\Sigma^{j}(K)$ and on $\Sigma_{d-j-1}(K)$, respectively; see [28] for an account of further preliminary results which aimed in the same direction.

The proof of Theorem 0.1 follows from the results concerning Hessian measures, established in the first part of the paper. Although a more direct argument along similar lines is possible, the present approach has the additional advantage of providing a unifying scheme. Hessian measures for convex and semi-convex functions were introduced as a counterpart to curvature measures for convex bodies (see [13] and [14]). In fact, the theory of Hessian 
measures now appears to be more general and flexible, since it yields the corresponding results for curvature and surface area measures by applying results for Hessian measures to special functions related to a convex body, like the distance function, the support function and the convex characteristic function. Moreover, an additional interesting and unexpected feature in the theory of Hessian measures, as presented here, is a notion of duality related to the conjugation of convex functions, for which no correspondence exists in the theory of support measures of convex bodies.

For a semi-convex function $u$ defined in an open convex subset $\Omega$ of $\mathbb{R}^{d}$, the Hessian measures $F_{j}(u, \cdot), j \in\{0,1, \ldots, d\}$, of $u$ can be obtained as coefficients of a local Steiner type formula (see the next section for precise definitions). More intuitively, Hessian measures generalize the integrals of the elementary symmetric functions of the eigenvalues of the Hessian matrix which is associated with $u$. We mention that in the papers [41] and [42] N. Trudinger and X. J. Wang introduced, following a different approach, the notion of Hessian measures in a certain class of functions, to study weak solutions of some elliptic partial differential equations. In the next section (see Remark 1.3), we observe that their definition coincides with ours, in the class of semi-convex functions. Still another approach to Hessian measures is implicitly contained in two papers by $\mathrm{Fu}$ [20], [21], where a general theory of Monge-Ampère functions is developed.

In the present paper we establish several new properties of Hessian measures. Our first result is a Crofton type formula, proved in Section 2, which is completely analogous to the classical Crofton formula for curvature measures of convex bodies (see [36], §4.5). An application of this formula is given in Section 4, in the proof of Theorem 4.2.

Sections 3 and 4 are devoted to the study of the absolute continuity of Hessian measures with respect to Hausdorff measures of suitable dimensions, and to find explicitly the corresponding Radon-Nikodym derivatives. More precisely, for a semi-convex function $u$, we prove that $F_{j}(u, \cdot)$ is absolutely continuous with respect to $\mathcal{H}^{j}$, for every $j \in\{0,1, \ldots, d\}$, and

$$
\left(\begin{array}{l}
d \\
j
\end{array}\right) F_{j}(u, \Gamma)=\int_{\Gamma} \mathcal{H}^{d-j}(\partial u(x)) d \mathcal{H}^{j}(x)
$$

for every Borel subset $\Gamma$ of $\Omega$ having $\sigma$-finite $j$-dimensional Hausdorff measure. Here again, similar to the case of convex bodies, the Hessian measures of $u$ depend on the singularities of $u$, which are measured in a quantitative way by Hausdorff measures of the subdifferential $\partial u$ of $u$.

Finally, in Section 5 , for a convex body $K \subset \mathbb{R}^{d}$, we prove various formulas relating the support measures of $K$ to the Hessian measures of the distance function, the convex characteristic function and the support function of $K$. As special cases of general results we find that the curvature measures of a set $X$ of positive reach are precisely the Hessian measures of the distance function $d_{X}$ of $X$; and for a convex body $K$, the surface area measures of $K$ are the Hessian measures of the support function of $K$. Using such formulas and the results proved for Hessian measures, we obtain the proof of Theorem 0.1 (see Theorem 5.5 and Theorem 5.11). 


\section{Hessian measures of semi-convex functions}

In this section we recall the definition of Hessian measures for semi-convex functions; furthermore we prove a result regarding the convergence, in the vague topology, of Hessian measures of a converging sequence of semi-convex functions.

For every $d \geq 1$, we denote by $\mathbb{R}^{d}$ the $d$-dimensional Euclidean space with scalar product $\langle\cdot, \cdot\rangle$ and norm $\|\cdot\|$. For every $k \in\{0,1, \ldots, d\}$, we denote by $\mathcal{H}^{k}$ the $k$-dimensional Hausdorff measure in $\mathbb{R}^{d}$.

If $\Omega$ is an open set in $\mathbb{R}^{d}$ and $\Omega^{\prime}$ is a convex open subset of $\Omega$ whose closure is a compact subset of $\Omega$, then we write $\Omega^{\prime} \subset \subset \Omega$.

Definition 1.1 Let $\Omega$ be an open subset of $\mathbb{R}^{d}$. A function $u: \Omega \rightarrow \mathbb{R}$ is said to be semi-convex in $\Omega$, if for every $\Omega^{\prime} \subset \subset \Omega$ there exists a finite constant $C \geq 0$ such that

$$
k(x):=u(x)+\frac{C}{2}\|x\|^{2}, \quad x \in \Omega^{\prime},
$$

defines a convex function in $\Omega^{\prime}$; the smallest such constant is denoted by $\operatorname{sc}\left(u, \Omega^{\prime}\right)$.

We will see in Section 5, Lemma 5.1, that the class of semi-convex functions thus defined coincides with the class of lower $C^{2}$-functions as defined and characterized in [35], Definition 10.29 and Theorem 10.33. Analytical properties of semi-convex functions, the connection between semi-convex functions and sets of positive reach, and applications of semi-convex (semi-concave) functions to solutions of Hamilton-Jacobi and Monge-Ampère equations, optimization theory or the calculus of variations have been the subject of a variety of papers and are of interest for research in various branches of mathematics; see for instance [32], [7], [8], [33], [19], [9], [20], [21], [31], [10], [2], [1], [5], [12], [44].

Note that semi-convex functions inherit all the regularity properties of convex functions, in particular they are locally Lipschitz. Consequently, if $u: \Omega \rightarrow \mathbb{R}$ is semi-convex, then at each point $x \in \Omega$ the Clarke (generalized) subgradient $\partial u(x)$ of $u$ is defined (see [11]).

Let $\Omega^{\prime} \subset \subset \Omega, C \geq \operatorname{sc}\left(u, \Omega^{\prime}\right)$ and $k(x):=u(x)+\frac{C}{2}\|x\|^{2}, x \in \Omega^{\prime}$; then, by basic properties of the subgradient, we have

$$
\partial u(x)=\partial k(x)-C x, \quad x \in \Omega^{\prime} .
$$

On the other hand, $\partial k$ coincides with the usual subgradient for convex functions, hence if $v \in \partial u(x)$ we get from $(1)$

$$
u(y) \geq u(x)+\langle y-x, v\rangle-\frac{C}{2}\|x-y\|^{2},
$$

for all $y \in \Omega^{\prime}$. Conversely, if $v \in \mathbb{R}^{d}$ is such that (2) is satisfied for some $C \geq 0$, then it is easy to see that $v \in \partial u(x)$.

Let $\Omega$ be an open subset of $\mathbb{R}^{d}$; in $[14$, Theorem 5.2] (see also [15, Theorem 2.1]), the present authors introduced, for every semi-convex function $u: \Omega \rightarrow \mathbb{R}$ and for every $\Omega^{\prime} \subset \subset$ 
$\Omega$, a sequence of $d+1$ signed Borel measures $F_{j}(u, \cdot), j \in\{0,1, \ldots, d\}$, over $\Omega^{\prime}$, which we will call the Hessian measures of $u$. This name is due to the fact that such measures generalize indefinite integrals of elementary symmetric functions of the eigenvalues of the Hessian matrix of $u$. Indeed if $u \in C^{2}(\Omega)$, then for every relatively compact, Borel measurable subset $\eta$ of $\Omega$,

$$
\left(\begin{array}{l}
d \\
k
\end{array}\right) F_{k}(u, \eta)=\int_{\eta} S_{d-k}\left(D^{2} u(x)\right) d \mathcal{H}^{d}(x), \quad k \in\{0,1, \ldots, d\},
$$

where $S_{j}\left(D^{2} u(x)\right)$ denotes the $j$-th elementary symmetric function of the eigenvalues of the Hessian matrix $D^{2} u(x)$ of $u$ at $x$, for every $j \in\{0,1, \ldots, d\}$.

For a general semi-convex function, the Hessian measures are the coefficients of a local Steiner type formula. In fact, for any Borel subset $\eta$ of $\Omega^{\prime}$ and $\rho \in\left[0, \operatorname{sc}\left(u, \Omega^{\prime}\right)^{-1}\right)$, consider the set

$$
P_{\rho}(u, \eta)=\{x+\rho v: x \in \eta, v \in \partial u(x)\},
$$

which is obtained by expanding $\eta$ along the subgradient of $u$. Then $P_{\rho}(u, \eta)$ is Borel measurable and

$$
\mathcal{H}^{d}\left(P_{\rho}(u, \eta)\right)=\sum_{k=0}^{d}\left(\begin{array}{l}
d \\
k
\end{array}\right) F_{k}(u, \eta) \rho^{d-k} .
$$

Remark 1.1 We note that the Hessian measures are independent of the subset $\Omega^{\prime} \subset \subset \Omega$ in the following sense: if $\Omega^{\prime}, \Omega^{\prime \prime} \subset \subset \Omega$, then the Hessian measures of $u$ corresponding to $\Omega^{\prime}$ and $\Omega^{\prime \prime}$ respectively, coincide on $\Omega^{\prime} \cap \Omega^{\prime \prime}$ (see [14, Remark 1 in Section 5]). In particular, the Hessian measures are well-defined for all Borel subsets of $\Omega$ whose closure is a compact subset of $\Omega$.

The main result of this section is the following theorem:

Theorem 1.1 Let $\Omega \subset \mathbb{R}^{d}$ be open, and let $u_{i}: \Omega \rightarrow \mathbb{R}, i \in \mathbb{N}$, be a sequence of semiconvex functions that converges pointwise on $\Omega$ to the semi-convex function $u: \Omega \rightarrow \mathbb{R}$. Assume that for each $x \in \Omega$ there is a neighbourhood $U \subset \subset \Omega$ of $x$ such that the sequence $\operatorname{sc}\left(u_{i}, U\right), i \in \mathbb{N}$, is bounded. Then, for every $k \in\{0,1, \ldots, d\}$, the sequence $F_{k}\left(u_{i}, \cdot\right)$, $i \in \mathbb{N}$, converges to $F_{k}(u, \cdot)$, with respect to the vague topology.

This result is in analogy to known properties of curvature measures of convex bodies, or more generally of sets of positive reach. Presently, we need such a theorem to prove a Crofton type formula for semi-convex functions.

For the proof we need some preliminary results.

Let $\Omega \subset \mathbb{R}^{d}$ be open, let $A$ be a subset of $\Omega$ and let $u: \Omega \rightarrow \mathbb{R}$ be a function; then we define

$$
\operatorname{lip}(u, A):=\sup \left\{\frac{|u(x)-u(y)|}{\|x-y\|}: x \neq y, x, y \in A\right\} .
$$

Further, we write $B(r)$ for a ball of radius $r \geq 0$ centred at the origin and $\bar{A}$ for the topological closure of a set $A \subset \mathbb{R}^{d}$. 
Lemma 1.2 Let $\Omega \subset \mathbb{R}^{d}$ be open, let $A \subset \subset \Omega$, and let $k: \Omega \rightarrow \mathbb{R}$ be convex. Then

$$
\operatorname{lip}(k, A)=\operatorname{lip}(k, \bar{A})=\sup \{\|v\|: v \in \partial k(x), x \in \bar{A}\} .
$$

Proof. Lebourg's mean value theorem for convex functions (see [11], Theorem 2.3.7) can be used for the non-trivial part.

Lemma 1.3 Let $\Omega \subset \mathbb{R}^{d}$ be open, let $A \subset \subset \Omega$, and let $k_{i}, k: \Omega \rightarrow \mathbb{R}, i \in \mathbb{N}$, be convex and such that $k_{i} \rightarrow k$ pointwise on $\Omega$ as $i \rightarrow \infty$. Then

$$
\lim _{i \rightarrow \infty} \operatorname{lip}\left(k_{i}, A\right)=\operatorname{lip}(k, A) \text {. }
$$

Proof. This follows, for example, from Lemma 1.2.

Lemma 1.4 Let $\Omega \subset \mathbb{R}^{d}$ be open, bounded and convex, let $A \subset \subset \Omega$, and let $k_{i}, k: \Omega \rightarrow \mathbb{R}$, $i \in \mathbb{N}$, be convex and such that $k_{i} \rightarrow k$ pointwise on $\Omega$ as $i \rightarrow \infty$. Let $R>0$ be such that $\Omega \subset B(R / 2)$. Then there exist convex Lipschitz functions $w_{i}, w: \mathbb{R}^{d} \rightarrow \mathbb{R}, i \in \mathbb{N}$, such that:

(i) $w_{i}, w$ are radially symmetric and of class $C^{\infty}$ on $\mathbb{R}^{d} \backslash B(R)$;

(ii) $w_{i} \rightarrow w$ pointwise on $\mathbb{R}^{d}$ as $i \rightarrow \infty,\left.w_{i}\right|_{A}=k_{i}$ for $i \in \mathbb{N}$ and $\left.w\right|_{A}=k$;

(iii) if $L_{i}:=\operatorname{lip}\left(w_{i}, \mathbb{R}^{d}\right), i \in \mathbb{N}$, and $L:=\operatorname{lip}\left(w, \mathbb{R}^{d}\right)$, then $L_{i} \rightarrow L$ as $i \rightarrow \infty$;

(iv) $P_{\rho}\left(w_{i}, B(2 R)\right)=B\left(2 R+\rho L_{i}\right)$ and $P_{\rho}(w, B(2 R))=B(2 R+\rho L)$ for all $\rho \geq 0$.

Proof. The proofs of parts (i), (ii) and (iv) follow from Lemmas 2.3, 2.4 and 2.5 in [13] and by the construction of approximating functions described there. Lemma 1.3 of the present paper can be used to prove (iii).

Proof of Theorem 1.1. We have to show that

$$
\lim _{i \rightarrow \infty} \int_{\Omega} f(x) d F_{k}\left(u_{i}, x\right)=\int_{\Omega} f(x) d F_{k}(u, x),
$$

for an arbitrary continuous function $f: \Omega \rightarrow \mathbb{R}$ with compact support in $\Omega$. Using a partition of unity, we see that we can assume that the support of $f$ is contained in an arbitrary prescribed set $A \subset \subset \Omega$. In particular, by the assumptions of the theorem, we can assume that there exists a constant $C \geq 0$ such that

$$
k(x):=u(x)+\frac{C}{2}\|x\|^{2}, \quad k_{i}(x):=u_{i}(x)+\frac{C}{2}\|x\|^{2}, \quad x \in A, i \in \mathbb{N},
$$

are convex in $\Omega$, and the sequence $k_{i}$ converges uniformly to $k$ in $A$. 
Let $w$ be any semi-convex function in $\Omega$ such that $\operatorname{sc}(w, A) \leq C$. Then from (4) we obtain, for every $r<1 / C$ and for every Borel subset $\eta$ of $A$,

$$
\left(\begin{array}{c}
\mathcal{H}^{d}\left(P_{\frac{1}{d+1} r}(w, \eta)\right) \\
\vdots \\
\mathcal{H}^{d}\left(P_{\frac{d+1}{d+1} r}(w, \eta)\right)
\end{array}\right)=M\left(\begin{array}{c}
\left(\begin{array}{l}
d \\
0
\end{array}\right) F_{d}(w, \eta) \\
\vdots \\
\left(\begin{array}{l}
d \\
d
\end{array}\right) F_{0}(w, \eta)
\end{array}\right)
$$

where

$$
M:=\left(\begin{array}{ccc}
\left(\frac{1}{d+1} r\right)^{0} & \cdots & \left(\frac{1}{d+1} r\right)^{d} \\
\vdots & & \vdots \\
\left(\frac{d+1}{d+1} r\right)^{0} & \cdots & \left(\frac{d+1}{d+1} r\right)^{d}
\end{array}\right) .
$$

The matrix $M$ is regular; let $\tilde{M}:=M^{-1}$ and

$$
\left(\tilde{a}_{i j}\right)_{\substack{i=0, \ldots, d \\ j=0, \ldots, d}}:=\tilde{M}
$$

Then we obtain the formula

$$
\left(\begin{array}{l}
d \\
i
\end{array}\right) F_{d-i}(w, \cdot)=\sum_{j=0}^{d} \tilde{a}_{i j} \mathcal{H}^{d}\left(P_{\frac{j+1}{d+1} r}(w, \cdot)\right),
$$

where the coefficients $\tilde{a}_{i j}$ do not depend on $w$, but only on $r$.

This argument shows that in order to prove the theorem it is sufficient to prove that $\mathcal{H}^{n}\left(P_{\rho}\left(u_{i}, \cdot\right)\right) \rightarrow \mathcal{H}^{n}\left(P_{\rho}(u, \cdot)\right)$ as $i \rightarrow \infty$, for any $\rho \in[0,1 / C)$, with respect to the vague topology on the space of Radon measures on the Borel subsets of $A$.

For $\rho \in[0,1 / C)$, we set $\mu_{\rho}(u, \cdot):=\mathcal{H}^{n}\left(P_{\rho}(u, \cdot)\right)$ and $\mu_{\rho}\left(u_{i}, \cdot\right):=\mathcal{H}^{n}\left(P_{\rho}\left(u_{i}, \cdot\right)\right), i \in \mathbb{N}$.

First, we prove that

$$
\limsup _{i \rightarrow \infty} \mu_{\rho}\left(u_{i}, \eta\right) \leq \mu_{\rho}(u, \eta)
$$

for compact sets $\eta \subset A$.

Let $\eta \subset A$ be a fixed compact set, and let $\rho \in[0,1 / C)$ be also fixed. By well-known properties of convex functions, the maps $k_{i}, i \in \mathbb{N}$, have a common Lipschitz constant $L^{\prime}$ on $\eta$, and hence, by (5), there is a common Lipschitz constant $L$, say, for the maps $u_{i}$, $i \in \mathbb{N}$, on $\eta$. Therefore

$$
P_{\rho}\left(u_{i}, \eta\right) \subset \eta+\rho B(L),
$$

and thus (6) follows from Fatou's theorem as soon as

$$
\limsup _{i \rightarrow \infty} P_{\rho}\left(u_{i}, \eta\right) \subset P_{\rho}(u, \eta)
$$

has been proved. To show $(7)$, let $y \in P_{\rho}\left(u_{i}, \eta\right)$ for all $i \in I$, where $I$ is an infinite subset of $\mathbb{N}$. Then $y=x_{i}+\rho v_{i}$ for some $x_{i} \in \eta$ and $v_{i} \in \partial u_{i}\left(x_{i}\right)$, for all $i \in I$. By the compactness of $\eta$ and by the existence of a common Lipschitz constant for $u_{i}, i \in \mathbb{N}$, there is an infinite 
subset $J \subset I$ such that $x_{i} \rightarrow x \in \eta$ and $v_{i} \rightarrow v$ as $i \rightarrow \infty$ and $i \in J$. By (5) and the characterization of subgradients in (2), it follows that $v \in \partial u(x)$, and hence $y=x+\rho v$, where $x \in \eta$ and $v \in \partial u(x)$. Thus (7) is established.

Next we show that

$$
\liminf _{i \rightarrow \infty} \mu_{\rho}\left(u_{i}, \eta\right) \geq \mu_{\rho}(u, \eta)
$$

for all open sets $\eta \subset A$ whose closure is a compact subset of $A$. Let such a set $\eta$ be fixed. By Fatou's theorem it is sufficient to show that

$$
P_{\rho}(u, \eta) \subset \liminf _{i \rightarrow \infty} P_{\rho}\left(u_{i}, \eta\right)
$$

Let $y \in P_{\rho}(u, \eta)$. Then $y=x+\rho v-\rho C x$, where $x \in \eta$ and $v \in \partial k(x)$, and hence $y^{\prime}:=x+\rho v \in P_{\rho}(k, \eta)$. Now we apply Lemma 1.4 to the sequence of convex functions $k_{i}$, $i \in \mathbb{N}, k$, and an open, convex and bounded neighbourhood of $A$ in $\Omega$. Using the notation of this lemma, we can write:

$$
y^{\prime} \in P_{\rho}(w, \eta) \subset \eta+\rho B(L) \subset B(R / 2)+B(\rho L) \subset B(R+\rho L) .
$$

Since $\rho, R$ are fixed, there is some $i_{0} \in \mathbb{N}$ such that

$$
P_{\rho}(w, \eta) \subset B\left(2 R+\rho L_{i}\right)=P_{\rho}\left(w_{i}, B(2 R)\right),
$$

for $i \geq i_{0}$. Here parts (iii) and (iv) of Lemma 1.4 were used. Therefore we can find $x_{i} \in B(2 R)$ and $v_{i} \in \partial w_{i}\left(x_{i}\right)$ such that $y^{\prime}=x_{i}+\rho v_{i}$. Using the compactness of $B(2 R)$, the fact that the sequence $\operatorname{lip}\left(w_{i}, A\right)$ is bounded and Theorem VI.6.2.7 in [30], we may assume that the sequences $x_{i}, v_{i}, i \in \mathbb{N}$, converge to $x_{0} \in B(2 R)$ and to $v \in \partial w\left(x_{0}\right)$, respectively; hence $y^{\prime}=x_{0}+\rho v_{0}$. On the other hand, $y^{\prime}=x+\rho v$ with $x \in \eta$ and $v \in \partial k(x)=\partial w(x)$. The uniqueness of this decomposition (with respect to $w$ ) yields that $x_{0}=x \in \eta$. Recall that $\eta$ is open. Hence, for $i \geq i_{1}\left(\geq i_{0}\right)$ we obtain that $x_{i} \in \eta$, and thus $y^{\prime}=x_{i}+\rho v_{i}, x_{i} \in \eta$, $v_{i} \in \partial w_{i}\left(x_{i}\right)=\partial k_{i}\left(x_{i}\right)$. This demonstrates that $y^{\prime} \in P_{\rho}\left(k_{i}, \eta\right)$, and then $y \in P_{\rho}\left(u_{i}, \eta\right)$, for $i \geq i_{1}$, that is

$$
y \in \liminf _{i \rightarrow \infty} P_{\rho}\left(u_{i}, \eta\right) .
$$

This yields (8) and thus completes the proof.

Lemma 1.5 Let $\Omega$ be an open subset of $\mathbb{R}^{d}$, let $u: \Omega \rightarrow \mathbb{R}$ be a semi-convex function, and let $\Omega^{\prime} \subset \subset \Omega$. Then there exists a sequence of $C^{\infty}$ functions $u_{i}: \Omega \rightarrow \mathbb{R}, i \in \mathbb{N}$, such that

(a) $u_{i} \rightarrow u$ pointwise on $\Omega^{\prime}$ as $i \rightarrow \infty$;

(b) $\operatorname{lip}\left(u_{i}, \Omega^{\prime}\right), \operatorname{sc}\left(u_{i}, \Omega^{\prime}\right), i \in \mathbb{N}$, are bounded sequences;

(c) $F_{k}\left(u_{i}, \cdot\right) \rightarrow F_{k}(u, \cdot)$ on $\Omega^{\prime}$ in the vague topology as $i \rightarrow \infty$, for $k \in\{0,1, \ldots, d\}$. 
Proof. Choose $\Omega^{\prime \prime} \subset \subset \Omega$ such that $\Omega^{\prime} \subset \subset \Omega^{\prime \prime}$. Then there exists a convex function $k: \Omega^{\prime \prime} \rightarrow \mathbb{R}$ and a constant $C \geq 0$ with

$$
u(x)=k(x)-\frac{C}{2}\|x\|^{2}, \quad x \in \Omega^{\prime \prime} .
$$

Lemma 2.3 in [13] applied to $\left.k\right|_{\Omega^{\prime}}$ shows that there is a convex and Lipschitz function $\tilde{k}: \mathbb{R}^{d} \rightarrow \mathbb{R}$ such that $\left.\tilde{k}\right|_{\Omega^{\prime}}=k$. By Lemma 2.4 in [13] we obtain a sequence of convex $C^{\infty}$ functions $k_{i}: \mathbb{R}^{d} \rightarrow \mathbb{R}, i \in \mathbb{N}$, which satisfy $k_{i}(x) \rightarrow \tilde{k}(x)=k(x)$ for all $x \in \Omega^{\prime}$. Now we define

$$
u_{i}(x):=k_{i}(x)-\frac{C}{2}\|x\|^{2}, \quad x \in \Omega .
$$

Then $u_{i}$ is of class $C^{\infty}$ and (a) is fulfilled. Moreover, (b) is implied by the properties of $k_{i}$ and the relative compactness of $\Omega^{\prime}$. Finally, (c) follows from Theorem 1.1.

Proposition 1.6 Let $\Omega$ be an open subset of $\mathbb{R}^{d}$, and let $u: \Omega \rightarrow \mathbb{R}$ be semi-convex. Further, let $C \in \mathbb{R}$ and let the semi-convex function $v: \Omega \rightarrow \mathbb{R}$ be defined by

$$
v(x):=u(x)+\frac{C}{2}\|x\|^{2}, \quad x \in \Omega .
$$

Then, for $k \in\{0,1, \ldots, d\}$ and for relatively compact, Borel measurable subsets $\eta$ of $\Omega$,

$$
F_{k}(u, \eta)=\sum_{j=0}^{d-k}\left(\begin{array}{c}
d-k \\
j
\end{array}\right)(-C)^{j} F_{k+j}(v, \eta) .
$$

Proof. From the expression of Hessian measures for smooth functions (see (3)) it follows immediately that (9) is true for every $u \in C^{2}(\Omega)$. Then the conclusion follows immediately from the preceding Lemma 1.5.

Remark 1.2 Proposition 1.6 is particularly useful when $v$ is a convex function. Since for any semi-convex function this can be assumed locally, equation (9) can be used for proving assertions about semi-convex functions by verifying corresponding results for convex functions only.

Remark 1.3 In the papers [41] and [42] N. Trudinger and X. J. Wang proved the existence of the first $k$ Hessian measures in the class of $k$-convex functions. Roughly speaking a function $u$ defined in an open subset $\Omega$ of $\mathbb{R}^{d}$ is $k$-convex, for some $k \in\{0,1, \ldots, d\}$, if it can be approximated pointwise by a decreasing sequence of functions $u_{i} \in C^{2}(\Omega), i \in \mathbb{N}$, such that $S_{j}\left(D^{2} u_{i}(x)\right) \geq 0$ for every $j \in\{0,1, \ldots, k\}, i \in \mathbb{N}$, and $x \in \Omega$. We notice that $d$-convexity is equivalent to the usual convexity.

Furthermore, in [42, Section 4] the notion of Hessian measures is extended to the class of $k$-semi-convex functions, i.e. the functions of the form $u(x)-C / 2\|x\|^{2}$, where 
$u$ is $k$-convex and $C \geq 0$ is a constant. Such an extension is performed as follows: if $v(x)=u(x)-C / 2\|x\|^{2}$, where $u$ is $k$-convex and $C \geq 0$, then the $k$-th Hessian measure $F_{k}(v, \cdot)$ of $v$ is defined through the Hessian measures of $u$ by the formula

$$
F_{k}(v, \cdot):=\sum_{j=0}^{k}\left(\begin{array}{c}
d-k \\
j
\end{array}\right)(-C)^{j} F_{k+j}(u, \cdot) .
$$

Thus, in particular, the definition of Hessian measures by Trudinger and Wang is given in a class which includes semi-convex functions; we notice that such a definition coincides with the one given in the present paper. (It should be observed that our measures $\left(\begin{array}{l}d \\ k\end{array}\right) F_{d-k}$ correspond to the measures $F_{k}$ in the notation of Trudinger and Wang.) Indeed by the above formula and by a special case of Proposition 1.6 it is enough to prove the equivalence of the two definitions in the class of convex functions. On the other hand in this class such an equivalence is straightforward since

1. the two definitions coincide for smooth convex functions;

2. both definitions require convergence of Hessian measures with respect to the vague topology, for approximating sequences of smooth convex functions.

\section{A Crofton formula for semi-convex functions}

We denote by $\mathrm{SO}(d)$ the topological group of proper rotations of $\mathbb{R}^{d}$, and by $\nu$ the Haar probability measure on $\mathrm{SO}(d)$. For every $d \geq 1$ and $k \in\{0,1, \ldots, d\}$, let $A(d, k)$ be the family of all $k$-dimensional affine subspaces of $\mathbb{R}^{d}$; following [36, §4.5] we construct an invariant measure $\mu_{k}$ over $A(d, k)$. Let $E_{0}$ be a fixed $k$-dimensional linear subspace, and denote by $E_{0}^{\perp}$ its orthogonal complement in $\mathbb{R}^{d}$. Consider the surjective map

$$
\gamma_{k}: E_{0}^{\perp} \times \mathrm{SO}(d) \rightarrow A(d, k), \quad \gamma(t, \rho):=\rho\left(E_{0}+t\right) .
$$

The set $A(d, k)$ is endowed with the finest topology such that $\gamma_{k}$ is continuous. Then the measure $\mu_{k}$ is defined as the image measure of the product measure $\lambda_{d-k} \otimes \nu$ under $\gamma_{k}$, defined on the Borel subsets of $E_{0}^{\perp} \times \mathrm{SO}(d)$, where $\lambda_{d-k}$ is the restriction of $\mathcal{H}^{d-k}$ to the Borel subsets of $E_{0}^{\perp}$. Thus $\mu_{k}$ is a Radon measure on the Borel sets of $A(d, k)$.

Let $\Omega$ be an open subset of $\mathbb{R}^{d}$, and let $u: \Omega \rightarrow \mathbb{R}$ be a semi-convex function. For $k \in\{0,1, \ldots, d\}$ and $E \in A(d, k)$, we denote by $\left.u\right|_{\Omega \cap E}$ the restriction of $u$ to $\Omega \cap E$. The function $\left.u\right|_{\Omega \cap E}$ is semi-convex on $\Omega \cap E$, hence its Hessian measures are defined; we denote them by $F_{j}^{(k)}\left(\left.u\right|_{\Omega \cap E}, \cdot\right), j \in\{0,1, \ldots, k\}$.

In this section we will prove the following:

Theorem 2.1 Let $\Omega$ be an open subset of $\mathbb{R}^{d}$, and let $u: \Omega \rightarrow \mathbb{R}$ be a semi-convex function. Further, let $k \in\{1,2, \ldots, d\}, j \in\{0,1, \ldots, k\}$, and let $\eta$ be a relatively compact, Borel measurable subset of $\Omega$. Then

$$
F_{d+j-k}(u, \eta)=\int_{A(d, k)} F_{j}^{(k)}\left(\left.u\right|_{\Omega \cap E}, \eta \cap E\right) d \mu_{k}(E) .
$$


In order to prove this theorem, we need two lemmas.

Lemma 2.2 Let $\Omega$ be an open subset of $\mathbb{R}^{d}$, and let $u: \Omega \rightarrow \mathbb{R}$ be semi-convex. If $\eta$ is a relatively compact, Borel measurable subset of $\Omega$, then the function

$$
g_{\eta}: A(d, k) \rightarrow \mathbb{R}, \quad g_{\eta}(E):=F_{j}^{(k)}\left(\left.u\right|_{\Omega \cap E}, \eta \cap E\right),
$$

is Borel measurable.

Proof. Let $\Omega^{\prime} \subset \subset \Omega$ be arbitrarily chosen. Using the argument described in the beginning of the proof of Theorem 1.1, we see that it is sufficient to prove that for every $\rho \in\left[0, \operatorname{sc}\left(u, \Omega^{\prime}\right)^{-1}\right)$ and for every Borel subset $\eta$ of $\Omega^{\prime}$ the function

$$
G_{\eta}: A(d, k) \rightarrow \mathbb{R}, \quad G_{\eta}(E):=\mathcal{H}^{k}\left(P_{\rho}^{(E)}\left(\left.u\right|_{\Omega \cap E}, \eta \cap E\right)\right),
$$

is Borel measurable, where

$$
P_{\rho}^{(E)}\left(\left.u\right|_{\Omega \cap E}, \eta \cap E\right):=\left\{x+\rho v: x \in \eta \cap E, v \in \partial\left(\left.u\right|_{\Omega \cap E}\right)\right\} .
$$

Notice that the function $G_{\eta}$ is nonnegative.

Let $\rho \in\left[0, \operatorname{sc}\left(u, \Omega^{\prime}\right)^{-1}\right)$ be fixed and let $\eta$ be a compact subset of $\Omega^{\prime}$. Since $u$ is Lipschitz on $\Omega^{\prime}$ and $G_{\eta}$ remains unchanged if $u$ is modified in the complement of $\Omega^{\prime}$, we can assume that $u$ is defined and semi-convex on $\mathbb{R}^{d}$ (see Lemma 2.3 in [13]).

Let $E, E_{i} \in A(d, k), i \in \mathbb{N}$, be such that $E_{i} \rightarrow E$ as $i \rightarrow \infty$. Then we can find $(t, \sigma),\left(t_{i}, \sigma_{i}\right) \in E_{0}^{\perp} \times \mathrm{SO}(d), i \in \mathbb{N}$, such that $E=\gamma_{k}(t, \sigma), E_{i}=\gamma_{k}\left(t_{i}, \sigma_{i}\right)$, for $i \in \mathbb{N}$, and $\left(t_{i}, \sigma_{i}\right) \rightarrow(t, \sigma)$ as $i \rightarrow \infty$. For every $i \in \mathbb{N}$, consider the isometry $\zeta_{i}: E \rightarrow E_{i}$ which is given by

$$
\zeta_{i}(x)=\sigma_{i}\left(\sigma^{-1}(x)-t+t_{i}\right), \quad x \in E,
$$

and the function

$$
v_{i}=\left.u\right|_{E_{i}} \circ \zeta_{i}=u \circ \zeta_{i},
$$

which is defined on $E$. Since $\zeta_{i}(x) \rightarrow x$ as $i \rightarrow \infty$ for all $x \in E$ and since $u$ is continuous, we obtain that $v_{i} \rightarrow u$ pointwise on $E$ as $i \rightarrow \infty$. Moreover, for any $U \subset \subset E$ there is some $U^{*} \subset \subset \mathbb{R}^{d}$ such that $\zeta_{i}(U) \subset U^{*}$ for all $i \in \mathbb{N}$. This implies that $\operatorname{sc}\left(v_{i}, U\right) \leq \operatorname{sc}\left(u, U^{*}\right)$ for all $i \in \mathbb{N}$. Therefore the sequence $\operatorname{sc}\left(v_{i}, U\right), i \in \mathbb{N}$, is bounded for any $U \subset \subset E$ and $v_{i}$ is semi-convex for all $i \in \mathbb{N}$. Hence Theorem 1.1 can be applied and we deduce that the sequence of measures $\mathcal{H}^{k}\left(P_{\rho}^{(E)}\left(\left.v_{i}\right|_{\Omega^{\prime} \cap E}, \cdot\right)\right), i \in \mathbb{N}$, converges in the vague topology to $\mathcal{H}^{k}\left(P_{\rho}^{(E)}\left(\left.u\right|_{\Omega^{\prime} \cap E}, \cdot\right)\right)$.

Set $\bar{\eta}_{i}:=\zeta_{i}^{-1}\left(\eta \cap E_{i}\right)$ and $\bar{\eta}:=\eta \cap E$. We have that

$$
P_{\rho}^{\left(E_{i}\right)}\left(\left.u\right|_{E_{i}}, \eta \cap E_{i}\right)=\zeta_{i}\left(P_{\rho}^{(E)}\left(v_{i}, \bar{\eta}_{i}\right)\right)
$$

and hence

$$
\mathcal{H}^{k}\left(P_{\rho}^{\left(E_{i}\right)}\left(\left.u\right|_{E_{i}}, \eta \cap E_{i}\right)\right)=\mathcal{H}^{k}\left(P_{\rho}^{(E)}\left(v_{i}, \bar{\eta}_{i}\right)\right)
$$


for all $i \in \mathbb{N}$. The sets $\bar{\eta}_{i}, i \in \mathbb{N}$, are compact subsets of $E$. For $\varepsilon>0$ we set

$$
\bar{\eta}_{\varepsilon}:=\{x \in E: \operatorname{dist}(x, \bar{\eta}) \leq \varepsilon\} .
$$

If $\varepsilon>0$ is sufficiently small, then $\bar{\eta}_{\varepsilon} \subset \Omega^{\prime} \cap E$; fix any such $\varepsilon>0$ for the moment. If $i \in \mathbb{N}$ is sufficiently large, then $\bar{\eta}_{i} \subset \bar{\eta}_{\varepsilon}$. Therefore

$$
\begin{aligned}
\mathcal{H}^{k}\left(P_{\rho}^{(E)}\left(\left.u\right|_{E}, \bar{\eta}_{\varepsilon}\right)\right) & \geq \limsup _{i \rightarrow \infty} \mathcal{H}^{k}\left(P_{\rho}^{(E)}\left(v_{i}, \bar{\eta}_{\varepsilon}\right)\right) \\
& \geq \limsup _{i \rightarrow \infty} \mathcal{H}^{k}\left(P_{\rho}^{(E)}\left(v_{i}, \bar{\eta}_{i}\right)\right) \\
& =\limsup _{i \rightarrow \infty} \mathcal{H}^{k}\left(P_{\rho}^{\left(E_{i}\right)}\left(\left.u\right|_{E_{i}}, \eta \cap E_{i}\right)\right) .
\end{aligned}
$$

On the other hand,

$$
\mathcal{H}^{k}\left(P_{\rho}^{(E)}\left(\left.u\right|_{E}, \eta \cap E\right)\right)=\inf _{\varepsilon>0} \mathcal{H}^{k}\left(P_{\rho}^{(E)}\left(\left.u\right|_{E}, \bar{\eta}_{\varepsilon}\right)\right)
$$

To see this, note that $\left.u\right|_{E}$ is Lipschitz in $\bar{\eta}_{\varepsilon}$ for $\varepsilon$ small enough, so that the set $P_{\rho}^{(E)}\left(\left.u\right|_{E}, \bar{\eta}_{\epsilon}\right)$ is bounded.

From (10) and (11) it follows that the function $G_{\eta}$ is upper semi-continuous, and hence measurable, whenever $\eta$ is a compact subset of $\Omega^{\prime}$.

Let $\mathcal{G}$ be the class of all sets $\eta \subset \Omega^{\prime}$ for which $G_{\eta}$ is Borel measurable. Obviously, $\mathcal{G}$ is a Dynkin system (see [6]) which contains the closed subsets of $\Omega^{\prime}$. Therefore $\mathcal{G}$ contains the Borel subsets of $\Omega^{\prime}$. But this immediately implies the assertion of the lemma.

If $u \in C^{2}(\Omega)$, where $\Omega$ is an open subset of $\mathbb{R}^{d}$, and $\left\{v_{1}, v_{2}, \ldots, v_{k}\right\}$ is any basis of the linear subspace parallel to $E \in A(d, k)$, then the Hessian matrix of $\left.u\right|_{\Omega \cap E}$ at $x \in \Omega \cap E$, with respect to the chosen basis, is given by

$$
D^{2}\left(\left.u\right|_{\Omega \cap E}\right)(x)=\left(\left\langle D^{2} u(x) v_{i}, v_{j}\right\rangle\right)_{i, j=1,2, \ldots, k} .
$$

Lemma 2.3 Let $\Omega$ be an open subset of $\mathbb{R}^{d}, u \in C^{2}(\Omega), k \in\{1,2, \ldots, d\}$, and $j \in$ $\{0,1, \ldots, k\}$. Then

$$
\int_{A(d, k)} \int_{\eta \cap E} S_{j}\left(D^{2}\left(\left.u\right|_{\Omega \cap E}\right)(x)\right) d \mathcal{H}^{k}(x) d \mu_{k}(E)=\beta_{d j k} \int_{\eta} S_{j}\left(D^{2} u(x)\right) d \mathcal{H}^{d}(x),
$$

where $\beta_{d j k}=\left(\begin{array}{l}d \\ k\end{array}\right)^{-1}\left(\begin{array}{l}d-j \\ k-j\end{array}\right)$.

Proof. Let $E_{0}$ be a $k$-dimensional linear subspace in $\mathbb{R}^{d}$, and let $E_{0}^{\perp}$ be the orthogonal complement of $E_{0}$ in $\mathbb{R}^{d}$. Taking into account the definition of the measure $\mu_{k}$, we can write

$$
\begin{aligned}
& \int_{A(d, k)} \int_{\eta \cap E} S_{j}\left(D^{2}\left(\left.u\right|_{\Omega \cap E}\right)(x)\right) d \mathcal{H}^{k}(x) d \mu_{k}(E) \\
& =\int_{\mathrm{SO}(d)} \int_{E_{0}^{\perp}} \int_{\eta \cap \rho\left(E_{0}+t\right)} S_{j}\left(D^{2}\left(\left.u\right|_{\Omega \cap \rho\left(E_{0}+t\right)}\right)(x)\right) d \mathcal{H}^{k}(x) d \mathcal{H}^{d-k}(t) d \nu(\rho) .
\end{aligned}
$$


Now let $\left\{v_{r}: r=1,2, \ldots, k\right\}$ be an orthonormal basis of $E_{0}$; for every $\rho \in \operatorname{SO}(d)$, $\left\{\rho\left(v_{r}\right): r=1,2, \ldots, k\right\}$ is an orthonormal basis of $\rho\left(E_{0}\right)$. Hence

$$
S_{j}\left(D^{2}\left(\left.u\right|_{\Omega \cap \rho\left(E_{0}+t\right)}\right)(x)\right)=S_{j}\left[\left(\left\langle D^{2} u(x) \rho\left(v_{i}\right), \rho\left(v_{j}\right)\right\rangle\right)_{i, j=1,2, \ldots, k}\right],
$$

for any $t \in E_{0}^{\perp}$ and $x \in \Omega \cap \rho\left(E_{0}+t\right)$. Substituting (13) into (12) and using Fubini's theorem, we obtain

$$
\begin{aligned}
& \int_{A(d, k)} \int_{\eta \cap E} S_{j}\left(D^{2}\left(\left.u\right|_{\Omega \cap E}\right)(x)\right) d \mathcal{H}^{k}(x) d \mu_{k}(E) \\
& =\int_{\mathrm{SO}(d)} \int_{\eta} S_{j}\left[\left(\left\langle D^{2} u(x) \rho\left(v_{i}\right), \rho\left(v_{j}\right)\right\rangle\right)_{i, j=1,2, \ldots, k}\right] d \mathcal{H}^{d}(x) d \nu(\rho) .
\end{aligned}
$$

The left-hand side of the preceding formula is independent of the choice of $E_{0}$. Let $\left\{v_{1}, v_{2}, \ldots, v_{d}\right\}$ be an orthonormal basis of $\mathbb{R}^{d}$. Fix $k$ indices $i_{1}, i_{2}, \ldots, i_{k} \in\{1,2, \ldots, d\}$ so that $1 \leq i_{1}<i_{2}<\cdots<i_{k} \leq d$, and let $E_{0}$ be the linear subspace spanned by $v_{i_{1}}, v_{i_{2}}, \ldots, v_{i_{k}}$. Then, repeating the above argument for every choice of the indices $i_{1}, i_{2}, \ldots, i_{k} \in\{1,2, \ldots, d\}$, and summing over all possible choices, we obtain

$$
\begin{aligned}
& \left(\begin{array}{l}
d \\
k
\end{array}\right) \int_{A(d, k)} \int_{\eta \cap E} S_{j}\left(D^{2}\left(\left.u\right|_{\Omega \cap E}\right)(x)\right) d \mathcal{H}^{k}(x) d \mu_{k}(E) \\
& =\int_{\mathrm{SO}(d)} \int_{\eta} \sum_{|I|=k} S_{j}\left[\left(\left\langle D^{2} u(x) \rho\left(v_{r}\right), \rho\left(v_{s}\right)\right\rangle\right)_{r, s \in I}\right] d \mathcal{H}^{d}(x) d \nu(\rho),
\end{aligned}
$$

where the summation is extended over all subsets $I \subset\{1, \ldots, d\}$ of cardinality $k$. On the other hand, it is readily seen that

$$
\sum_{|I|=k} S_{j}\left[\left(\left\langle D^{2} u(x) \rho\left(v_{r}\right), \rho\left(v_{s}\right)\right\rangle\right)_{r, s \in I}\right]=\left(\begin{array}{l}
d-j \\
k-j
\end{array}\right) S_{j}\left(D^{2} u(x)\right) .
$$

To complete the proof, it is sufficient to insert (15) into (14).

Proof of Theorem 2.1. It is sufficient to consider the case where $\Omega^{\prime} \subset \subset \Omega$ and $\eta$ is a Borel measurable subset of $\Omega^{\prime}$.

Step 1. Assume that $u \in C^{2}(\Omega)$, fix a Borel subset $\eta$ of $\Omega^{\prime}, k \in\{1,2, \ldots, d\}$ and $j \in\{0,1, \ldots, k\}$. By (3) and the previous lemma, we obtain

$$
\begin{aligned}
\left(\begin{array}{l}
k \\
j
\end{array}\right)\left(\begin{array}{l}
d \\
k
\end{array}\right) F_{d+j-k}(u, \eta) & =\left(\begin{array}{c}
d-k+j \\
j
\end{array}\right) \int_{\eta} S_{k-j}\left(D^{2} u(x)\right) d \mathcal{H}^{d}(x) \\
& =\left(\begin{array}{l}
d \\
k
\end{array}\right) \int_{A(d, k)} \int_{\eta \cap E} S_{k-j}\left(D^{2}\left(\left.u\right|_{\Omega \cap E}\right)(x)\right) d \mathcal{H}^{k}(x) d \mu_{k}(E) \\
& =\left(\begin{array}{l}
k \\
j
\end{array}\right)\left(\begin{array}{l}
d \\
k
\end{array}\right) \int_{A(d, k)} F_{j}^{(k)}\left(\left.u\right|_{\Omega \cap E}, \eta \cap E\right) d \mu_{k}(E) .
\end{aligned}
$$


Hence it follows that the theorem is true for $C^{2}$ functions. Moreover, for any continuous function $f: \mathbb{R}^{d} \rightarrow \mathbb{R}$ with compact support contained in $\Omega^{\prime}$, we deduce

$$
\int_{\Omega} f(x) d F_{d+j-k}(u, x)=\int_{A(d, k)} \int_{\Omega \cap E} f(x) d F_{j}^{(k)}\left(\left.u\right|_{\Omega \cap E}, x\right) d \mu_{k}(E) .
$$

In order to prove the theorem, we have to show that (16) is true for an arbitrary semi-convex function $u$ on $\Omega$.

Step 2. By Lemma 1.5 there exists a sequence of semi-convex functions $u_{i}: \Omega \rightarrow \mathbb{R}$, $u_{i} \in C^{\infty}(\Omega), i \in \mathbb{N}$, converging pointwise to $u$ on $\Omega^{\prime}$ and such that

$$
\operatorname{lip}\left(u_{i}, \Omega^{\prime}\right), \operatorname{sc}\left(u_{i}, \Omega^{\prime}\right) \leq C,
$$

for some positive constant $C$ and for all $i \in \mathbb{N}$.

Let $f: \mathbb{R}^{d} \rightarrow \mathbb{R}$ be a continuous function with compact support contained $\Omega^{\prime}$. Theorem 1.1 yields

$$
\lim _{i \rightarrow \infty} \int_{\Omega^{\prime}} f(x) d F_{d+j-k}\left(u_{i}, x\right)=\int_{\Omega^{\prime}} f(x) d F_{d+j-k}(u, x) .
$$

Furthermore, for every $E \in A(d, k)$ the sequence $\left.u_{i}\right|_{\Omega^{\prime} \cap E}, i \in \mathbb{N}$, converges pointwise to $\left.u\right|_{\Omega^{\prime} \cap E}$, and by (17) the sequence $\operatorname{sc}\left(\left.u_{i}\right|_{\Omega^{\prime} \cap E}, \Omega^{\prime} \cap E\right), i \in \mathbb{N}$, is bounded. Another application of Theorem 1.1 thus yields

$$
\lim _{i \rightarrow \infty} \int_{\Omega^{\prime} \cap E} f(x) d F_{j}^{(k)}\left(\left.u_{i}\right|_{\Omega^{\prime} \cap E}, x\right)=\int_{\Omega^{\prime} \cap E} f(x) d F_{j}^{(k)}\left(\left.u\right|_{\Omega^{\prime} \cap E}, x\right),
$$

for every $E \in A(d, k)$.

Now taking into account that the functions $u_{i}$ are uniformly Lipschitz in $\Omega^{\prime}$ (by (17)), that $\Omega^{\prime}$ is bounded, and using Proposition 1.6 of the present paper and Theorem 6.2 in [14], it is possible to prove that there exists a constant $K>0$, depending on $C, \operatorname{diam}\left(\Omega^{\prime}\right)$, $k$ and $d$, such that

$$
\int_{\Omega^{\prime} \cap E} f(x) d F_{j}^{(k)}\left(\left.u_{i}\right|_{\Omega^{\prime} \cap E}, x\right) \leq K\|f\|_{\infty},
$$

for every $i \in \mathbb{N}$ and for every $E \in A(d, k)$. Hence we can apply the dominated convergence theorem to obtain

$$
\begin{aligned}
\lim _{i \rightarrow \infty} \int_{A(d, k)} & \int_{\Omega^{\prime} \cap E} f(x) d F_{j}^{(k)}\left(\left.u_{i}\right|_{\Omega^{\prime} \cap E}, x\right) d \mu_{k}(E) \\
= & \int_{A(d, k)} \int_{\Omega^{\prime} \cap E} f(x) d F_{j}^{(k)}\left(\left.u\right|_{\Omega^{\prime} \cap E}, x\right) d \mu_{k}(E) .
\end{aligned}
$$

The proof can now be completed by combining (18), (19) and the result of step 1. 


\section{Absolute continuity of Hessian measures}

In this section we study the absolute continuity of the $k$-th Hessian measure of a semiconvex function with respect to the $k$-dimensional Hausdorff measure $\mathcal{H}^{k}$. Recall that for two Borel measures $\mu, \nu$ over $\mathbb{R}^{d}$ one defines that $\mu$ is absolutely continuous with respect to $\nu$ if $\nu(A)=0$ implies $\mu(A)=0$ for all Borel sets $A \subset \mathbb{R}^{d}$. If this is the case and if the restriction $\nu\left\llcorner B\right.$ of $\nu$ to a fixed Borel set $B \subset \mathbb{R}^{d}$ is $\sigma$-finite, then it follows by the abstract Radon-Nikodym theorem of measure theory that $\mu\llcorner B$ has a density with respect to $\nu\llcorner B$. The explicit determination of this density in the present framework is the subject of Section 4.

We wish to emphasize already at this point that the results and remarks of this section almost immediately lead to corresponding results for curvature and surface area measures, as soon as the results of Section 5 are available.

The crucial result in this section is the following theorem; its proof is based on an idea which was applied in the context of curvature measures by H. Fallert [16].

Theorem 3.1 Let $\Omega$ be an open subset of $\mathbb{R}^{d}$, and let $u: \Omega \rightarrow \mathbb{R}$ be a semi-convex function. Then, for every $k \in\{0,1, \ldots, d\}$, the $k$-th Hessian measure of $u, F_{k}(u, \cdot)$, is absolutely continuous with respect to $\mathcal{H}^{k}$ over $\Omega$.

Proof. A decomposition argument shows that it is sufficient to consider Borel subsets $\eta$ of a set $\Omega^{\prime} \subset \subset \Omega$. Moreover, by virtue of Proposition 1.6, it suffices to prove the theorem in the special case where $u$ is convex in $\Omega^{\prime}$.

We denote by $\Theta_{j}(u, \cdot), j \in\{0,1, \ldots, d\}$ the support measures whose existence is proved in $\left[14\right.$, Theorem 3.1]. We recall that $\Theta_{j}(u, \cdot)$, for every $j \in\{0,1, \ldots, d\}$, is a nonnegative Borel measure over $\Omega^{\prime} \times \mathbb{R}^{d}$ and

$$
F_{j}(u, \eta)=\Theta_{j}\left(u, \eta \times \mathbb{R}^{d}\right)
$$

for every Borel subset $\eta$ of $\Omega^{\prime}$.

Let $i \in\{0,1, \ldots, d\}$ and let $\eta \subset \Omega^{\prime}$ be a Borel set which satisfies $\mathcal{H}^{d-i}(\eta)=0$. From (20) it follows that it is sufficient to prove that $\Theta_{d-i}(u, \eta \times B(L))=0$ for every $L>0$. Let $z \in \mathbb{R}^{d}$ and $r>0$ be arbitrarily chosen. We write $B(z, r)$ for the ball of radius $r$ centred at $z$. Fix an arbitrary $L>0$. Taking into account the defining relation for the measures $\Theta_{j}(u, \cdot)$ (see [14, Theorem 3.1]), we may argue as in the beginning of the proof of Theorem 1.1 and thus obtain

$$
\left(\begin{array}{l}
d \\
i
\end{array}\right) \Theta_{d-i}(u,(B(z, r) \cap \eta) \times B(L))=\sum_{j=0}^{d} \tilde{a}_{i j} \mathcal{H}^{d}\left(P_{\frac{j+1}{d+1} r}(u,(B(z, r) \cap \eta) \times B(L))\right)
$$

where the coefficients $\tilde{a}_{i j}$ are such that the matrix

$$
\tilde{M}:=\left(\tilde{a}_{i j}\right)_{\substack{i=0, \ldots, d \\ j=0, \ldots, d}}
$$


is the inverse of

$$
M:=\left(\begin{array}{ccc}
\left(\frac{1}{d+1} r\right)^{0} & \ldots & \left(\frac{1}{d+1} r\right)^{d} \\
\vdots & & \vdots \\
\left(\frac{d+1}{d+1} r\right)^{0} & \cdots & \left(\frac{d+1}{d+1} r\right)^{d}
\end{array}\right) .
$$

All constants which appear in the following will be independent of $r$. Since

$$
\begin{aligned}
\tilde{a}_{i j} & =\frac{(-1)^{i+j} \operatorname{det}\left(\left(a_{k l}\right)_{\substack{k \neq j \\
l \neq i}}\right)}{\operatorname{det} M}, \\
\operatorname{det} M & =\left(r^{0} r^{1} \cdots r^{d}\right) c(d)=r^{\frac{d(d+1)}{2}} c(d),
\end{aligned}
$$

with a constant $c(d) \neq 0$, and

$$
\operatorname{det}\left(\left(a_{k l}\right)_{\substack{k \neq j \\ l \neq i}}\right)=c_{1}(d, i, j)\left(r^{0} r^{1} \cdots r^{d}\right) r^{-i}=c_{1}(d, i, j) r^{\frac{d(d+1)}{2}-i},
$$

with a constant $c_{1}(d, i, j)$, we finally obtain

$$
\tilde{a}_{i j}=c_{2}(d, i, j) r^{-i},
$$

with a constant $c_{2}(d, i, j)$. In addition, we have

$$
\begin{aligned}
\mathcal{H}^{d}\left(P_{\frac{j+1}{d+1} r}(u,(B(z, r) \cap \eta) \times B(L))\right) & \leq \mathcal{H}^{d}\left(B(z, r)+\frac{j+1}{d+1} r B(L)\right) \\
& \leq(1+L)^{d} r^{d} \alpha(d) .
\end{aligned}
$$

From (21), (22) and (23) we conclude that

$$
\Theta_{d-i}(u,(B(z, r) \cap \eta) \times B(L)) \leq c_{1}(d, i)(1+L)^{d} r^{d-i},
$$

with a constant $c_{1}(d, i)$.

Now let $\eta \subset \Omega^{\prime}$ be an arbitrary Borel set, and let $\delta>0$ be fixed. Choose $z_{l} \in \mathbb{R}^{d}$ and $r_{l} \in(0, \delta], l \in \mathbb{N}$, in such a way that

$$
\eta \subset \bigcup_{l \in \mathbb{N}} B\left(z_{l}, r_{l}\right)
$$

Then we deduce

$$
\begin{aligned}
\Theta_{d-i}(u, \eta \times B(L)) & \leq \sum_{l \in \mathbb{N}} \Theta_{d-i}\left(u,\left(B\left(z_{l}, r_{l}\right) \cap \eta\right) \times B(L)\right) \\
& \leq c_{1}(d, i)(1+L)^{d} \sum_{l \in \mathbb{N}} r_{l}^{d-i} \\
& =\frac{c_{1}(d, i)}{\alpha(d-i)}(1+L)^{d} \sum_{l \in \mathbb{N}} \alpha(d-i)\left(\frac{\operatorname{diam} B\left(z_{l}, r_{l}\right)}{2}\right)^{d-i},
\end{aligned}
$$


and hence

$$
\begin{aligned}
\Theta_{d-i}(u, \eta \times B(L)) & \leq c_{2}(d, i)(1+L)^{d} \mathcal{S}_{\delta}^{d-i}(\eta) \\
& \leq c_{2}(d, i)(1+L)^{d} \mathcal{S}^{d-i}(\eta) \\
& \leq c_{2}(d, i)(1+L)^{d} 2^{d-i} \mathcal{H}^{d-i}(\eta)=0
\end{aligned}
$$

where $c_{2}(d, i)$ is a constant and $\mathcal{S}^{d-i}$ denotes the $(d-i)$-dimensional spherical measure over $\mathbb{R}^{d}$ (see [18], Section 2.10.2). This yields the desired conclusion.

Remark 3.1 The construction of Hessian measures, which was carried out for finite convex functions in [14], immediately extends to (proper) closed convex functions defined on $\mathbb{R}^{d}$ which take values in $\overline{\mathbb{R}}$ (confer Section 5). Furthermore for such functions the same proof as in the above theorem can be repeated. Hence if $u: \mathbb{R}^{d} \rightarrow \overline{\mathbb{R}}$ is closed and convex, then the $j$-th Hessian measure $F_{j}(u, \cdot)$ of $u$ is absolutely continuous with respect to $\mathcal{H}^{j}$ in $\mathbb{R}^{d}$, for every $j \in\{0,1, \ldots, d\}$.

Remark 3.2 For convex Lipschitz functions which take values in $\mathbb{R}$ a more precise result than Theorem 3.1 can be proved by a different approach. Let $\Omega$ be an open convex subset of $\mathbb{R}^{d}$, let $u: \Omega \rightarrow \mathbb{R}$ be a convex Lipschitz function with Lipschitz constant $L$, and let $j \in\{0,1, \ldots, d\}$. Then

$$
F_{j}(u, \cdot) \leq c(d, j) L^{d-j} \mathcal{H}^{j}
$$

where $c(d, j)=\left(\frac{2 d}{d+1}\right)^{\frac{j}{2}} \frac{\alpha(d)}{\alpha(j)}$. This fact can be proved as follows. Let $z \in \mathbb{R}^{d}$ and $r \geq 0$ be such that $B(z, r) \subset \Omega$. Then Theorem 6.2 in [14] yields

$$
F_{j}(u, B(z, r)) \leq L^{d-j} W_{d-j}(B(z, r))=L^{d-j} r^{j} \alpha(d),
$$

where the quantities $W_{j}(B(z, r)), j \in\{0,1, \ldots, d\}$, denote the quermassintegrals of $B(z, r)$. The remaining part of the proof is a repetition of the proof for Theorem 3.1.

\section{Radon-Nikodym derivatives of Hessian measures}

Let $\Omega$ be an open convex subset of $\mathbb{R}^{d}$ and let $u: \Omega \rightarrow \mathbb{R}$ be semi-convex. In the previous section we have seen that the $j$-th Hessian measure $F_{j}(u, \cdot)$ of $u$ is absolutely continuous with respect to the measure $\mathcal{H}^{j}$, for every $j \in\{0,1, \ldots, d\}$. In the present section we compute the Radon-Nikodym derivative of $F_{j}(u, \cdot)$ with respect to $\mathcal{H}^{j}$ on subsets of $\Omega$ of $\sigma$-finite $j$-dimensional Hausdorff measure. Thus we obtain a generalization of Theorem 5.3 in [14], since the set $\Sigma^{j}(u), j \in\{0,1, \ldots, d\}$, of $j$-singular points of $u$ (see the definition in the proof of Theorem 4.2) is countably $j$-rectifiable and hence has $\sigma$-finite $j$-dimensional Hausdorff measure.

In order to determine the Radon-Nikodym derivative we need a lemma. 
Lemma 4.1 Let $\Omega \subset \mathbb{R}^{d}$ be open, $x \in \Omega$, let $u: \Omega \rightarrow \mathbb{R}$ be semi-convex, and let $x \in E \in$ $A(d, d-j)$ for some $j \in\{0,1, \ldots, d-1\}$. Then

$$
\operatorname{dim} \partial\left(\left.u\right|_{\Omega \cap E}\right)(x) \leq \operatorname{dim} \partial u(x) .
$$

Proof. Equation (1) shows that it is sufficient to prove the assertion for convex functions. Furthermore, by the interplay between convex functions and their epigraphs, the assertion follows from the following geometric statement. Let $K \subset \mathbb{R}^{d}$ be a closed convex set, let $E \in A(d, k)$ for some $k \in\{1, \ldots, d\}$, and assume that $E \cap$ int $K \neq \emptyset$. Let $L(E)$ denote the linear subspace parallel to $E$, and let $x \in E \cap \partial K$. Then

$$
N_{E}(K \cap E, x)=N(K, x) \mid L(E),
$$

where $N_{E}(K \cap E, x)$ is the normal cone of $K \cap E$ at $x$ taken in $E$ and $N(K, x) \mid L(E)$ is the orthogonal projection of the normal cone of $K$ at $x$ onto $L(E)$. Further, let $S(K, x)$ denote the support cone of $K$ at $x$ and let $S_{E}(K \cap E, x)$ denote the support cone of $K \cap E$ at $x$ (calculated in $E$ ). Using notation and results as described in Section 2.2 of [36], we obtain

$$
\begin{aligned}
N_{E}(K \cap E, x) & =\left(S_{E}(K \cap E, x)\right)^{*}=(S(K, x) \cap L(E))^{*}=S(K, x)^{*} \mid L(E) \\
& =N(K, x) \mid L(E),
\end{aligned}
$$

where the first and the second formation of polar cones is taken in $L(E)$.

Theorem 4.2 Let $\Omega$ be an open subset of $\mathbb{R}^{d}$, and let $u: \Omega \rightarrow \mathbb{R}$ be semi-convex. Let $j \in\{0,1, \ldots, d\}$, and let $\Gamma \subset \Omega$ be a Borel set having $\sigma$-finite $j$-dimensional Hausdorff measure. Then

$$
\left(\begin{array}{l}
d \\
j
\end{array}\right) F_{j}(u, \Gamma)=\int_{\Gamma} \mathcal{H}^{d-j}(\partial u(x)) d \mathcal{H}^{j}(x) .
$$

Proof. For the proof we can assume that $\Omega^{\prime} \subset \subset \Omega$ and $\Gamma \subset \Omega^{\prime}$ has finite $j$-dimensional Hausdorff measure.

For $k \in\{0,1, \ldots, d\}$ we define the set of singular points of order $k$ of $u$ by

$$
\Sigma^{k}(u):=\{x \in \Omega: \operatorname{dim}(\partial u(x)) \geq d-k\} .
$$

In $[14$, Theorem 5.3] it is proved that

$$
\left(\begin{array}{l}
d \\
j
\end{array}\right) F_{j}\left(u, \Gamma \cap \Sigma^{j}(u)\right)=\int_{\Gamma \cap \Sigma^{j}(u)} \mathcal{H}^{d-j}(\partial u(x)) d \mathcal{H}^{j}(x),
$$

and hence, by the definition of $\Sigma^{j}(u)$,

$$
\left(\begin{array}{l}
d \\
j
\end{array}\right) F_{j}\left(u, \Gamma \cap \Sigma^{j}(u)\right)=\int_{\Gamma} \mathcal{H}^{d-j}(\partial u(x)) d \mathcal{H}^{j}(x) .
$$


It remains to prove that $F_{j}\left(u, \Gamma \backslash \Sigma^{j}(u)\right)=0$.

By [18, Section 3.2.14] $\Gamma$ can be split into two disjoint Borel sets, $\Gamma=\Gamma_{r} \cup \Gamma_{u}$, where $\Gamma_{r}$ is countably $j$-rectifiable, and $\Gamma_{u}$ is purely $\left(\mathcal{H}^{j}, j\right)$-unrectifiable. Set $\Gamma_{r}^{j}:=\Gamma_{r} \backslash \Sigma^{j}(u)$ and $\Gamma_{u}^{j}:=\Gamma_{u} \backslash \Sigma^{j}(u)$.

Let us prove that

$$
F_{j}\left(u, \Gamma_{r}^{j}\right)=0 .
$$

A special case of Theorem 2.1 yields

$$
F_{j}\left(u, \Gamma_{r}^{j}\right)=\int_{A(d, d-j)} F_{0}^{(d-j)}\left(\left.u\right|_{\Omega \cap E}, \Gamma_{r}^{j} \cap E\right) d \mu_{d-j}(E)
$$

Since $\Gamma_{r}^{j}$ is $\left(\mathcal{H}^{j}, j\right)$-rectifiable, Theorem 3.3.13 and Section 2.10.16 in [18] yield, for $\mu_{d-j}$ almost every $E \in A(d, d-j)$, that $E \cap \Gamma_{r}^{j}$ is a finite set. Hence

$$
F_{j}\left(u, \Gamma_{r}^{j}\right)=\int_{A^{\prime}} F_{0}^{(d-j)}\left(\left.u\right|_{\Omega \cap E}, \Gamma_{r}^{j} \cap E\right) d \mu_{d-j}(E)
$$

where $A^{\prime}$ is the set of all $E \in A(d, d-j)$ for which $\Gamma_{r}^{j} \cap E$ is non-empty and finite.

Now let $E \in A^{\prime}$, and $\Gamma_{r}^{j} \cap E=\left\{x_{1}, x_{2}, \ldots, x_{p}\right\}$. Since $\operatorname{dim}\left(\partial u\left(x_{i}\right)\right)<d-j$ for all $i \in\{1, \ldots, p\}$, and since $F_{0}^{(d-j)}\left(\left.u\right|_{\Omega \cap E},\left\{x_{i}\right\}\right)$ is the $(d-j)$-dimensional measure of the image set of $\left\{x_{i}\right\}$ through the subgradient of $\left.u\right|_{\Omega \cap E}$ (see [13, Theorem 5.3]), Lemma 4.1 implies

$$
F_{0}^{(d-j)}\left(\left.u\right|_{\Omega \cap E},\left\{x_{i}\right\}\right)=0, \quad i=1,2, \ldots, p
$$

and then

$$
F_{0}^{(d-j)}\left(\left.u\right|_{\Omega \cap E}, \Gamma_{r}^{j} \cap E\right)=0 .
$$

Using (28), which holds for every $E \in A^{\prime}$, and (27), we get (25).

Now using again formula (26) with $\Gamma_{r}^{j}$ replaced by $\Gamma_{u}^{j}$, and [18, Theorem 3.3 .13 and Section 2.10.16], we obtain that

$$
F_{j}\left(u, \Gamma_{u}^{j}\right)=0
$$

which completes the proof.

Remark 4.1 It is a remarkable consequence of Theorem 4.2 that the restriction of the signed Hessian measure $F_{j}(u, \cdot)$ of a semi-convex function $u$ to sets having $\sigma$-finite $j$ dimensional Hausdorff measure yields a nonnegative measure. Moreover, the proof of the preceding theorem shows that Theorem 5.3 in [14] can be generalized in the same way. This fact will be used in the following section. We finally remark that in view of Proposition 1.6 it would be sufficient to prove Theorem 4.2 for convex functions. 


\section{Applications to support measures}

In this section we shall explain the basic relationship between Hessian measures of semiconvex functions and support measures of sets of positive reach. In [14], the epigraph of a semi-convex function (which is a set of positive reach) was used in a non-trivial way to construct the Hessian measures of the given function. Now we shall see that support measures of a set with positive reach can be expressed in a simple way as Hessian measures of some particular functions related to the given set, such as the distance function, support function and convex characteristic function.

Before we go into the details of the results, we recall some basic notions and introduce some notations regarding sets of positive reach and convex bodies in $\mathbb{R}^{d}$. For a subset $X$ of $\mathbb{R}^{d}$ and for $r \geq 0$ we denote by $X_{r}$ the set of points whose distance from $X$ is $r$ at the most. The reach of $X$, reach $(X)$, is the supremum over all $r \geq 0$ such that for every point $x \in X_{r}$ there exists a unique nearest point in $X$ to $x$. We say that $X \subset \mathbb{R}^{d}$ is a set of positive reach (has positive reach) if $\operatorname{reach}(X)>0$.

A convex body $K$ in $\mathbb{R}^{d}$, i.e. a convex compact subset of $\mathbb{R}^{d}$ with non-empty interior, is clearly a set of positive reach and $\operatorname{reach}(K)=\infty$.

Let $X \subset \mathbb{R}^{d}$ have positive reach; for $r<\operatorname{reach}(X)$ and $x \in X_{r} \backslash X$ we write $p(X, x)$ for the nearest point in $X$ to $x$, and we set $u(X, x):=d_{X}(x)^{-1}(x-p(X, x))$, where $d_{X}(x)$ is the distance of $x$ to $X$. Furthermore for every $x \in X$, we denote by $N(X, x)$ the normal cone of $X$ at $x$ (see [17]); the definition given there is consistent with the one adopted in convex geometry. Also recall that if $X$ is a convex body and $u \in S^{d-1}$, then

$$
F(X, u):=\{x \in X: u \in N(X, x)\}
$$

is the support set of $X$ in direction $u$.

The support measures $\Theta_{j}(X, \cdot), j=0,1, \ldots, d-1$, of a set $X \subset \mathbb{R}^{d}$ of positive reach are signed measures defined for all bounded Borel subsets of $\mathbb{R}^{d} \times S^{d-1}$. They can be obtained as coefficients of the local Steiner formula

$$
\mathcal{H}^{d}\left(M_{r}(X, \eta)\right)=\frac{1}{d} \sum_{j=1}^{d-1} r^{d-j}\left(\begin{array}{l}
d \\
r
\end{array}\right) \Theta_{j}(X, \eta),
$$

where $0<r<\operatorname{reach}(X), \eta$ is a bounded Borel subset of $\mathbb{R}^{d} \times S^{d-1}$ and

$$
M_{r}(X, \eta):=\left\{x \in X_{r} \backslash X:(p(X, x), u(X, x)) \in \eta\right\} .
$$

By specializing $\eta$ we obtain the Federer curvature measures (briefly: curvature measures) of $X$ as follows

$$
C_{j}(X, \alpha):=\Theta_{j}\left(X, \alpha \times S^{d-1}\right), \quad j \in\{0,1, \ldots, d-1\},
$$

for every bounded Borel set $\alpha \subset \mathbb{R}^{d}$. Moreover in the special case where $X=K$ is a convex body we can also introduce the surface area measures of $K$ by setting

$$
S_{j}(K, \beta):=\Theta_{j}\left(K, \mathbb{R}^{d} \times \beta\right), \quad j \in\{0,1, \ldots, d-1\},
$$


for every Borel subset $\beta$ of $S^{d-1}$.

Now we are in the position to show some applications of the results proved in the previuos sections to support measures of sets of positive reach. We start with a preliminary lemma which shows that we could have chosen a seemingly weaker definition of semiconvexity in the first instance. We include this simple lemma, because the method of proof will also be useful in the following.

Lemma 5.1 Let $\Omega \subset \mathbb{R}^{d}$ be open, and let $u: \Omega \rightarrow \mathbb{R}$ be a function. Then $u$ is semi-convex if and only if for each $x \in \Omega$ there exists a neighbourhood $U \subset \subset \Omega$ of $x$ and a constant $C \geq 0$ such that

$$
k(x):=u(x)+\frac{C}{2}\|x\|^{2}, \quad x \in U,
$$

defines a convex function.

Proof. We prove the "if part". Let $\Omega^{\prime} \subset \subset \Omega$ be given. Then there are neighbourhoods $U_{1}, \ldots, U_{p} \subset \Omega$ and constants $C_{1}, \ldots, C_{p} \geq 0$ such that

$$
k_{i}(x):=u(x)+\frac{C_{i}}{2}\|x\|^{2}, \quad x \in U_{i},
$$

for $i=1, \ldots, p$, defines a convex function. We set

$$
C:=\max \left\{C_{i}: 1 \leq i \leq p\right\}
$$

and

$$
k(x):=u(x)+\frac{C}{2}\|x\|^{2}, \quad x \in \Omega^{\prime} .
$$

Obviously, $\left.k\right|_{U_{i}}$ is a convex function for each $i \in\{1, \ldots, p\}$, and therefore the epigraph of each of these functions is an open convex set. But then the epigraph of $\left.k\right|_{\Omega^{\prime}}$ fulfills the local support property which is required for an application of Tietze's convexity criterion; see [43], Theorem 4.10. Thus $\left.k\right|_{\Omega^{\prime}}$ is a convex function.

In the more general context of Riemannian geometry, a less explicit version of the following proposition has been proved by Kleinjohann [33], Satz 2.8; see also [8] and [19], Corollary 3.4, for a converse.

Proposition 5.2 Let $X \subset \mathbb{R}^{d}$ satisfy reach $(X)>r>0$ and let $0<\delta<r$. Then $d_{X}$ is semi-convex on int $X_{r-\delta}$; in particular, $\operatorname{sc}\left(d_{X}, \Omega^{\prime}\right) \leq \delta^{-1}$ for all $\Omega^{\prime} \subset \subset$ int $X_{r-\delta}$.

Proof. We show that

$$
k(x):=d_{X}(x)+\frac{1}{2 \delta}\|x\|^{2}, \quad x \in \mathbb{R}^{d},
$$

defines a convex function on convex subsets of int $X_{r-\delta}$. In view of the proof of Lemma 5.1 we have to show that for each $x_{0} \in \operatorname{int} X_{r-\delta}$ there is some $r_{0}>0$ such that the epigraph of $\left.k\right|_{B\left(x_{0}, r_{0}\right)}$ is supported by a hyperplane at $\left(x_{0}, k\left(x_{0}\right)\right)$. 
If $x_{0} \in X$, then the support property is satisfied since $d_{X} \geq 0$ and $x \mapsto(2 \delta)^{-1}\|x\|^{2}$ is convex. Now let $x_{0} \in\left(\right.$ int $\left.X_{r-\delta}\right) \backslash X$. We define $p_{0}:=p\left(X, x_{0}\right), u_{0}:=u\left(X, x_{0}\right)$ and $r_{0}:=2^{-1} \min \left\{d_{X}\left(x_{0}\right), r-\delta-d_{X}\left(x_{0}\right)\right\}$, and hence we obtain

$$
B\left(x_{0}, r_{0}\right) \subset B\left(p_{0}+r u_{0}, r\right) \backslash B\left(p_{0}+r u_{0}, \delta\right) .
$$

Furthermore, we define $C:=\mathbb{R}^{d} \backslash$ int $B\left(p_{0}+r u_{0}, r\right)$; a straightforward calculation then shows that

$$
x \mapsto d_{C}(x)+\frac{1}{2 \delta}\|x\|^{2}
$$

defines a convex function on $B\left(x_{0}, r_{0}\right)$. Therefore we find some (uniquely determined) vector $v \in \mathbb{R}^{d}$ such that

$$
d_{C}\left(x_{0}+h\right)+\frac{1}{2 \delta}\left\|x_{0}+h\right\|^{2}-d_{C}\left(x_{0}\right)-\frac{1}{2 \delta}\left\|x_{0}\right\|^{2} \geq\langle v, h\rangle
$$

for all $h \in \mathbb{R}^{d}$ with $\|h\|<r_{0}$. Since $d_{C}\left(x_{0}\right)=d_{X}\left(x_{0}\right)$ and $d_{C}\left(x_{0}+h\right) \leq d_{X}\left(x_{0}+h\right)$ for $h \in \mathbb{R}^{d}$ with $\|h\|<r_{0}$, we obtain

$$
d_{X}\left(x_{0}+h\right)+\frac{1}{2 \delta}\left\|x_{0}+h\right\|^{2}-d_{X}\left(x_{0}\right)-\frac{1}{2 \delta}\left\|x_{0}\right\|^{2} \geq\langle v, h\rangle,
$$

for $\|h\|<r_{0}$. Thus the epigraph of $x \mapsto d_{X}(x)+(2 \delta)^{-1}\|x\|^{2}$ locally has support planes, which was to be shown.

Remark 5.1 Instead of sets of positive reach one can consider the more general sets having the unique footpoint property (UFP-sets); see [33] and [8]. These are closed sets $X \subset \mathbb{R}^{d}$ for which $\operatorname{reach}(X, x)>0$ for all $x \in X$. Recall from [17] that $\operatorname{reach}(X, \cdot)$ is a continous function on $X$, and therefore compact UFP-sets are sets of positive reach. The method of proof for Proposition 5.2 can be used to show that the distance function of a closed set having the unique footpoint property is semi-convex in $U(X)$, where

$$
U(X):=\bigcup_{x \in X} \operatorname{int} B(x, \operatorname{reach}(X, x))
$$

is an open neighbourhood of $X$. In [33] this was proved in Riemannian spaces by using $H$-Jacobi fields. We wish to point out that Proposition 5.3 and Theorems 5.4 and 5.5 could also be stated for UPF-sets, since all relevant notions are locally defined.

Our next purpose is to describe the subdifferential of the distance function of a set $X$, satisfying reach $(X)>r$, in terms of geometric quantities. The result can be paraphrased by saying that sets of positive reach are regular in the sense of nonsmooth analysis.

Proposition 5.3 Let $X \subset \mathbb{R}^{d}$ satisfy $\operatorname{reach}(X)>r>0$. Then

$$
\partial d_{X}(x)= \begin{cases}N(X, x) \cap B(0,1), & x \in X, \\ \{u(X, x)\}, & x \notin X_{r} \backslash X .\end{cases}
$$


Proof. The case $x \notin X$ is covered by Theorem 4.8 (3) and (5) in [17].

Now let $x \in X$. Consider sequences $x_{i} \in X, v_{i} \in \mathbb{R}^{d} \backslash\{0\}, i \in \mathbb{N}$, such that $x_{i} \rightarrow x$, $v_{i} \rightarrow 0$ and $v_{i} /\left\|v_{i}\right\| \rightarrow v \in \mathbb{R}^{d}$ as $i \rightarrow \infty$ and such that $p\left(X, x_{i}+v_{i}\right)=x_{i}$ for all $i \in \mathbb{N}$. Then $v_{i} \in N\left(X, x_{i}\right)$ and therefore $x_{i}=p\left(X, x_{i}+r v_{i} /\left\|v_{i}\right\|\right)$; see [17], Theorem 4.8. Since $x_{i}+r v_{i} /\left\|v_{i}\right\| \rightarrow x+r v$ as $i \rightarrow \infty$ and since $p(X, \cdot)$ is continuous on $X_{r}$, we deduce that $x=p(X, x+r v)$, and hence $v \in N(X, x)$. But then [11], Theorem 2.5.6, implies that $\partial d_{X}(x) \subset N(X, x) \cap B(0,1)$, since $N(X, x) \cap B(0,1)$ is convex.

Conversely, let $v \in N(X, x) \cap B(0,1)$ for some $x \in X$. Since $0 \in \partial d_{X}(x)$ (note that $d_{X}(x)=0$ and $d_{X} \geq 0$ ), we can assume that $v \neq 0$. Then we have to show that

$$
\limsup _{\substack{y \rightarrow x \\ t \downarrow 0}} \frac{d_{X}(y+t w)-d_{X}(y)}{t} \geq\langle v, w\rangle,
$$

for all $w \in \mathbb{R}^{d}$. We prove that

$$
\limsup _{t \downarrow 0} \frac{d_{X}(x+t w)-d_{X}(x)}{t} \geq\langle v, w\rangle
$$

for all $w \in \mathbb{R}^{d}$, from which (29) follows. If $\langle v, w\rangle \leq 0$, then there is nothing to prove; hence we assume that $\langle v, w\rangle>0$. But then $x+t w \in B\left(x+r\|v\|^{-1} v, r\right) \subset\left(\mathbb{R}^{d} \backslash X\right) \cup\{x\}$, if $t>0$ is sufficiently small, which implies

$$
\frac{d_{X}(x+t w)}{t} \geq \frac{r-\|-r\| v\left\|^{-1} v+t w\right\|}{t} .
$$

Passing to the limit, we obtain

$$
\limsup _{t \downarrow 0} \frac{d_{X}(x+t w)}{t} \geq\left\langle\frac{v}{\|v\|}, w\right\rangle \geq\langle v, w\rangle,
$$

since $\|v\| \in(0,1]$.

The following theorem describes the surprisingly simple connection between the support measures of a set of positive reach and the Hessian measures of the distance function $d_{X}$ of $X$.

Theorem 5.4 Let $X \subset \mathbb{R}^{d}$ have positive reach, let $\alpha \subset X$ be relatively compact and Borel measurable, let $\beta \subset S^{d-1}$ be Borel measurable, and set $\hat{\beta}:=\operatorname{conv}(\beta \cup\{0\})$. Then

$$
\Theta_{j}(X, \alpha \times \beta)=d \Theta_{j}\left(d_{X}, \alpha \times \hat{\beta}\right), \quad j \in\{0,1, \ldots, d-1\} .
$$

Proof. Let reach $(X)>r>0$ and let $U \subset \subset$ int $X_{r / 2}$ be arbitrarily fixed. By Proposition 5.2 we obtain $\operatorname{sc}\left(d_{X}, U\right) \leq 2 / r$, and hence

$$
\mathcal{H}^{d}\left(P_{\rho}\left(d_{X}, \gamma \times \hat{\beta}\right)\right)=\sum_{j=0}^{d} \rho^{d-j}\left(\begin{array}{l}
d \\
j
\end{array}\right) \Theta_{j}\left(d_{X}, \gamma \times \hat{\beta}\right)
$$


holds for $\rho \in[0, r / 2)$ and Borel sets $\gamma \subset U$; see Theorem 5.2 in [14]. For $\gamma \subset U \cap X$ and $\rho>0$ we also have

$$
\begin{aligned}
P_{\rho}\left(d_{X}, \gamma \times \hat{\beta}\right) & =\left\{x+\rho p \in \mathbb{R}^{d}: x \in \gamma, p \in \hat{\beta} \cap \partial d_{X}(x)\right\} \\
& =(\gamma \cap \operatorname{int} X) \cup\left\{x+\rho p \in \mathbb{R}^{d}: x \in \gamma \cap \partial X, p \in \hat{\beta} \cap N(X, x)\right\} \\
& =(\gamma \cap X) \cup M_{\rho}(X, \gamma \times \beta),
\end{aligned}
$$

where Theorem 4.8 (12) in [17] was used. Hence the Steiner formula for sets with positive reach yields, for a Borel set $\gamma \subset U \cap X$ and $0<\rho<r / 2$, that we also have

$$
\mathcal{H}^{d}\left(P_{\rho}\left(d_{X}, \gamma \times \hat{\beta}\right)\right)=\mathcal{H}^{d}(\gamma \cap X)+\frac{1}{d} \sum_{j=0}^{d-1} \rho^{d-j}\left(\begin{array}{l}
d \\
j
\end{array}\right) \Theta_{j}(X, \gamma \times \hat{\beta})
$$

A comparison of coefficients in (30) and (31) yields the assertion, first for Borel sets $\gamma \subset U$, and then in the general case by additivity.

The preceding theorem can be used to deduce results about support measures of a set with positive reach from corresponding results about Hessian measures of the distance function of the given set. As a particular example we establish a generalization of Theorem 3.2 in [28].

Theorem 5.5 Let $X \subset \mathbb{R}^{d}$ be a set with positive reach, and let $j \in\{0,1, \ldots, d-1\}$. Further, let $\alpha \subset \mathbb{R}^{d}$ be a Borel set having $\sigma$-finite $j$-dimensional Hausdorff measure, and let $\eta \subset \alpha \times S^{d-1}$ be Borel measurable. Then

$$
\left(\begin{array}{c}
d-1 \\
j
\end{array}\right) \Theta_{j}(X, \eta)=\int_{\mathbb{R}^{d}} \mathcal{H}^{d-1-j}\left(N(X, x) \cap \eta_{x}\right) d \mathcal{H}^{j}(x),
$$

where $\eta_{x}:=\left\{u \in S^{d-1}:(x, u) \in \eta\right\}$.

Proof. It is sufficient to prove the asserted equality for $\eta=\gamma \times \beta$, where $\gamma \subset \alpha$ and $\beta \subset$ $S^{d-1}$ are Borel sets and $\gamma$ is bounded. Recall that $\Theta_{j}(X, \cdot)$ is concentrated on $\partial X \times S^{d-1}$, and by Proposition 5.3 we have $\partial d_{X}(x) \cap \hat{\beta}=N(X, x) \cap \hat{\beta}$ if $x \in \partial X$. Using Theorems 5.4 
and 4.2 together with Remark 4.1, we can conclude

$$
\begin{aligned}
\left(\begin{array}{c}
d-1 \\
j
\end{array}\right) \Theta_{j}(X, \gamma \times \beta) & =\left(\begin{array}{c}
d-1 \\
j
\end{array}\right) \Theta_{j}(X,(\gamma \cap \partial X) \times \beta) \\
& =d\left(\begin{array}{c}
d-1 \\
j
\end{array}\right) \Theta_{j}\left(d_{X},(\gamma \cap \partial X) \times \hat{\beta}\right) \\
& =(d-j) \int_{\gamma \cap \partial X} \mathcal{H}^{d-j}\left(\partial d_{X}(x) \cap \hat{\beta}\right) d \mathcal{H}^{j}(x) \\
& =(d-j) \int_{\gamma \cap \partial X} \mathcal{H}^{d-j}(N(X, x) \cap \hat{\beta}) d \mathcal{H}^{j}(x) \\
& =\int_{\gamma \cap \partial X} \mathcal{H}^{d-1-j}(N(X, x) \cap \beta) d \mathcal{H}^{j}(x) \\
& =\int_{\mathbb{R}^{d}} \mathcal{H}^{d-1-j}\left(N(X, x) \cap(\gamma \times \beta)_{x}\right) d \mathcal{H}^{j}(x) .
\end{aligned}
$$

In order to justify the fifth equality it is sufficient to check that

$$
(d-j) \mathcal{H}^{d-j}(N(X, x) \cap \hat{\beta})=\mathcal{H}^{d-1-j}(N(X, x) \cap \beta)
$$

for all $x \in \partial X$ for which $\operatorname{dim} N(X, x)=d-j$.

Subsequently, we study convex functions which may take infinite values and whose epigraph is a closed set. We can assume that such a function is defined in $\mathbb{R}^{d}$, since we may extend a function $u: \Omega \rightarrow \overline{\mathbb{R}}, \Omega \subset \mathbb{R}^{d}$ non-empty, open and convex, by setting $\left.u\right|_{\mathbb{R}^{d} \backslash \Omega}: \equiv \infty$.

The theory developed in [14] extends to (proper) closed convex functions $u: \mathbb{R}^{d} \rightarrow \overline{\mathbb{R}}$. The only change concerns the definition of the normal bundle of $u$ in $\mathbb{R}^{d+1} \times \mathbb{R}^{d+1}$, which is now given by

$$
\operatorname{Nor}(u):=\left\{(X, V) \in \operatorname{Nor}(\operatorname{epi}(u)):\left\langle V, E_{n+1}\right\rangle \neq 0\right\}
$$

see [14] for the notation and further details. We should also emphasize that if $p \in \partial u(x)$, then necessarily $u(x)<\infty$.

For a closed convex set $K \subset \mathbb{R}^{d}$, let $\mathbf{I}_{K}$ denote the convex characteristic function of $K$,

$$
\mathbf{I}_{K}(x):= \begin{cases}0, & \text { if } x \in K \\ \infty, & \text { if } x \notin K\end{cases}
$$

The following lemma is easy to check from the definitions.

Lemma 5.6 Let $K \subset \mathbb{R}^{d}$ be a closed convex set. Then

$$
\partial \mathbf{I}_{K}(x)= \begin{cases}N(K, x), & x \in K, \\ \emptyset, & x \notin K .\end{cases}
$$


From Lemma 5.6 we can now derive a representation for the support measures of a convex set in terms of the Hessian measures of the convex characteristic function which is associated with the set.

Theorem 5.7 Let $K \subset \mathbb{R}^{d}$ be closed and convex. Let $\alpha \subset \mathbb{R}^{d}$ and $\beta \subset S^{d-1}$ be Borel measurable. Then

$$
\Theta_{j}(K, \alpha \times \beta)=d \Theta_{j}\left(\mathbf{I}_{K}, \alpha \times \hat{\beta}\right), \quad j \in\{0,1, \ldots, d-1\} .
$$

Proof. For any $\rho>0$ we obtain

$$
\begin{aligned}
P_{\rho}\left(\mathbf{I}_{K}, \alpha \times \hat{\beta}\right) & =\left\{x+\rho p \in \mathbb{R}^{d}: x \in \alpha, p \in \partial \mathbf{I}_{K}(x) \cap \hat{\beta}\right\} \\
& =(\alpha \cap \operatorname{int} K) \cup\left\{x+\rho p \in \mathbb{R}^{d}: x \in \alpha \cap \partial K, p \in N(K, x) \cap \hat{\beta}\right\} \\
& =(\alpha \cap K) \cup M_{\rho}(K, \alpha \times \beta) .
\end{aligned}
$$

By an application of the Steiner formulae for convex functions and convex sets, we conclude

$$
\sum_{j=0}^{d} \rho^{d-j}\left(\begin{array}{l}
d \\
j
\end{array}\right) \Theta_{j}\left(\mathbf{I}_{K}, \alpha \times \hat{\beta}\right)=\frac{1}{d} \sum_{m=0}^{d-1} \rho^{d-m}\left(\begin{array}{c}
d \\
m
\end{array}\right) \Theta_{m}(K, \alpha \times \beta)+\mathcal{H}^{d}(\alpha \cap K) .
$$

A comparison of coefficients yields the desired result.

For a closed convex function $u: \mathbb{R}^{d} \rightarrow \overline{\mathbb{R}}$ the conjugate function is defined by

$$
u^{*}(y):=\sup \left\{\langle y, x\rangle-u(x): x \in \mathbb{R}^{d}\right\}, \quad y \in \mathbb{R}^{d} .
$$

The conjugate function $u^{*}$ is again closed and convex and $u^{* *}=u$. In a certain (but very vague) sense, the formation of the polar body of a given convex set can be seen in analogy to the conjugation of convex functions. For the following theorem, however, a corresponding result for convex sets is not true. On the other hand, in spherical space an analogous theorem has been proved for spherical support measures and pairs of polar (spherically) convex sets; see Glasauer [25].

Theorem 5.8 Let $u: \mathbb{R}^{d} \rightarrow \overline{\mathbb{R}}$ be closed and convex, and let $\alpha, \beta \subset \mathbb{R}^{d}$ be Borel measurable. Then

$$
\Theta_{j}(u, \alpha \times \beta)=\Theta_{d-j}\left(u^{*}, \beta \times \alpha\right), \quad j \in\{0,1, \ldots, d\} .
$$

Proof. By [34], Theorem 23.5, we obtain for any $\rho>0$ that

$$
\begin{aligned}
P_{\rho}(u, \alpha \times \beta) & =\left\{x+\rho p \in \mathbb{R}^{d}: x \in \alpha, p \in \beta, p \in \partial u(x)\right\} \\
& =\rho\left\{p+\rho^{-1} x \in \mathbb{R}^{d}: p \in \beta, x \in \alpha, x \in \partial u^{*}(p)\right\} \\
& =\rho P_{\rho^{-1}}\left(u^{*}, \beta \times \alpha\right) .
\end{aligned}
$$


The Steiner formula for convex functions, Theorem 3.1 in [14], now yields

$$
\begin{aligned}
\sum_{j=0}^{d} \rho^{d-j}\left(\begin{array}{l}
d \\
j
\end{array}\right) \Theta_{j}(u, \alpha \times \beta) & =\rho^{d} \mathcal{H}^{d}\left(P_{\rho^{-1}}\left(u^{*}, \beta \times \alpha\right)\right) \\
& =\rho^{d} \sum_{j=0}^{d} \rho^{j-d}\left(\begin{array}{l}
d \\
j
\end{array}\right) \Theta_{j}\left(u^{*}, \beta \times \alpha\right) \\
& =\sum_{j=0}^{d} \rho^{j}\left(\begin{array}{l}
d \\
j
\end{array}\right) \Theta_{j}\left(u^{*}, \beta \times \alpha\right) .
\end{aligned}
$$

A comparison of coefficients completes the proof.

For a compact convex set $K \subset \mathbb{R}^{d}$ we denote by $h_{K}$ the support function of $K$; see [36].

Corollary 5.9 Let $K \subset \mathbb{R}^{d}$ be a compact convex set, and let $\alpha \subset \mathbb{R}^{d}, \beta \subset S^{d-1}$ be Borel measurable. Then

$$
\Theta_{j}(K, \alpha \times \beta)=d \Theta_{d-j}\left(h_{K}, \hat{\beta} \times \alpha\right), \quad j \in\{0,1, \ldots, d-1\} .
$$

Proof. Use $\left(h_{K}\right)^{*}=\mathbf{I}_{K}$ and Theorems 5.7 and 5.8.

In particular, the following special cases of Theorem 5.4 and Corollary 5.9 deserve to be emphasized.

Corollary 5.10 Let $X \subset \mathbb{R}^{d}$ be a set of positive reach, let $K \subset \mathbb{R}^{d}$ be a compact convex set, let $\alpha \subset \mathbb{R}^{d}, \beta \subset S^{d-1}$ be Borel sets, and assume that $\alpha$ is bounded. Then

$$
C_{j}(X, \alpha)=d F_{j}\left(d_{X}, \alpha \cap \partial X\right), \quad j \in\{0,1, \ldots, d-1\},
$$

and

$$
S_{j}(K, \beta)=d F_{d-j}\left(h_{K}, \hat{\beta}\right), \quad j \in\{0,1, \ldots, d-1\} .
$$

Our final result improves Theorem 4.3 in [28] in the same way as Theorem 5.5 generalizes Theorem 3.2 in [28].

Theorem 5.11 Let $K \subset \mathbb{R}^{d}$ be a convex body, and let $j \in\{0,1, \ldots, d-1\}$. Further, let $\omega \subset S^{d-1}$ be a Borel set having $\sigma$-finite $(d-1-j)$-dimensional Hausdorff measure, and let $\eta \subset \mathbb{R}^{d} \times \omega$ be Borel measurable. Then

$$
\left(\begin{array}{c}
d-1 \\
j
\end{array}\right) \Theta_{j}(K, \eta)=\int_{S^{d-1}} \mathcal{H}^{j}\left(F(K, u) \cap \eta^{u}\right) d \mathcal{H}^{d-1-j}(u),
$$

where $\eta^{u}:=\left\{x \in \mathbb{R}^{d}:(x, u) \in \eta\right\}$. 
Proof. It is sufficient to consider the case where $\eta=\gamma \times \beta$ with Borel sets $\gamma \subset \mathbb{R}^{d}$ and $\beta \subset \omega$. We can assume that $\gamma \subset K$. First, we show that $\hat{\beta}$ has $\sigma$-finite $(d-j)$ dimensional Hausdorff measure. To see this, we assume that $\mathcal{H}^{d-1-j}(\beta)<\infty$ and deduce that $\mathcal{H}^{d-j}(\hat{\beta})<\infty$. By Theorem 2.10.45 in [18], we obtain $\mathcal{H}^{d-j}([0,1] \times \beta)<\infty$. The map $f:[0,1] \times S^{d-1} \rightarrow \mathbb{R}^{d},(\lambda, u) \mapsto \lambda u$, is Lipschitz and hence

$$
\mathcal{H}^{d-j}(\hat{\beta})=\mathcal{H}^{d-j}(f([0,1] \times \beta)) \leq(\operatorname{lip}(f))^{d-j} \mathcal{H}^{d-j}([0,1] \times \beta)<\infty
$$

Using Corollary 5.9 and Theorem 4.2 together with Remark 4.1, we obtain

$$
\begin{aligned}
\left(\begin{array}{c}
d-1 \\
j
\end{array}\right) \Theta_{j}(K, \gamma \times \beta) & =d\left(\begin{array}{c}
d-1 \\
j
\end{array}\right) \Theta_{d-j}\left(h_{K}, \hat{\beta} \times \gamma\right) \\
& =(d-j) \int_{\hat{\beta}} \mathcal{H}^{j}\left(\partial h_{K}(x) \cap \gamma\right) d \mathcal{H}^{d-j}(x) \\
& =(d-j) \int_{\left(\beta \cap \Sigma_{d-j-1}(K)\right)^{\wedge}} \mathcal{H}^{j}\left(\partial h_{K}(x) \cap \gamma\right) d \mathcal{H}^{d-j}(x) \\
& =\int_{\beta \cap \Sigma_{d-j-1}(K)} \mathcal{H}^{j}(F(K, u) \cap \gamma) d \mathcal{H}^{d-1-j}(u) \\
& =\int_{S^{d-1}} \mathcal{H}^{j}\left(F(K, u) \cap(\gamma \times \beta)^{u}\right) d \mathcal{H}^{d-1-j}(u),
\end{aligned}
$$

where

$$
\Sigma_{r}(K)=\left\{u \in S^{d-1}: \operatorname{dim} F(K, u) \geq d-1-r\right\}
$$

is an $r$-rectifiable Borel set, for $r \in\{0,1, \ldots, d-1\}$; therefore the fourth equality can be justified by the area/coarea theorem [18].

\section{References}

[1] G. Alberti, On the structure of singular sets of convex functions, Calc. Var. Partial Differential Equations 2 (1994), 17-27.

[2] G. Alberti, L. Ambrosio and P. Cannarsa, On the singularities of convex functions, Manuscripta Math. 76 (1992), 421-435.

[3] S. Alesker, Description of continuous isometry covariant valuations on convex sets, Geom. Dedicata 74 (1999), 241-248.

[4] S. Alesker, Continuous rotation invariant valuations on convex sets, to appear in Ann. of Math. 
[5] G. Anzellotti and E. Ossanna, Singular sets of convex bodies and surfaces with generalized curvatures, Manuscripta Math. 86 (1995), 417-433.

[6] R. B. Ash, Measure, Integration and Functional Analysis, Academic Press, New York, 1972.

[7] V. Bangert, Analytische Eigenschaften konvexer Funktionen auf Riemannschen Mannigfaltigkeiten, J. Reine Angew. Math. 307/308 (1979), 309-324.

[8] V. Bangert, Sets with positive reach, Arch. Math. 38 (1982), 54-57.

[9] A. Canino, On p-convex sets and geodesics, J. Differential Equations 75 (1988), 118157.

[10] A. Canino, Local properties of geodesics on p-convex sets, Ann. Mat. Pura Appl. 49 (1991), 17-44.

[11] F. H. Clarke, Optimization and Nonsmooth Analysis, Canadian Mathematical Society, Wiley-Interscience Publication, New York, 1983.

[12] F. H. Clarke, R. J. Stern and P. R. Wolenski, Proximal smoothness and the lower-C ${ }^{2}$ property, J. Convex Anal. 2 (1995), 117-144.

[13] A. Colesanti, A Steiner type formula for convex functions, Mathematika 44 (1997), 195-214.

[14] A. Colesanti and D. Hug, Steiner type formulae and weighted measures of singularities for semi-convex functions, to appear in Trans. Amer. Math. Soc.

[15] A. Colesanti and P. Salani, Generalised solutions of Hessian equations, Bull. Austral. Math. Soc. 56 (1997), 459-466.

[16] H. Fallert, Quermaßdichten für Punktprozesse konvexer Körper und Boolesche Modelle, Math. Nachr. 181 (1996), 165-184.

[17] H. Federer, Curvature measures, Trans. Amer. Math. Soc. 93 (1959), 418-491.

[18] H. Federer, Geometric Measure Theory, Springer, Berlin, 1969.

[19] J. H. G. Fu, Tubular neighborhoods in Euclidean spaces, Duke Math. J. 52 (1985), 1025-1046.

[20] J. H. G. Fu, Monge-Ampère functions, I, Indiana Univ. Math. J. 38 (1989), 745-771.

[21] J. H. G. Fu, Monge-Ampère functions, II, Indiana Univ. Math. J. 38 (1989), 773-789.

[22] R. J. Gardner, Geometric Tomography, Cambridge University Press, Cambridge, 1995.

[23] A. Giannopoulos and M. Papadimitrakis, Isotropic surface area measures, Preprint. 
[24] A. A. Giannopoulos and V. D. Milman, Extremal problems and isotropic positions of convex bodies, Preprint.

[25] S. Glasauer, Integralgeometrie konvexer Körper im sphärischen Raum, Ph.D. Thesis, Freiburg, 1995.

[26] P. Goodey, E. Lutwak and W. Weil, Functional analytic characterizations of classes of convex bodies, Math. Z. 222 (1996), 363-381.

[27] P. Goodey and W. Weil, Zonoids and generalizations, in Handbook of convex geometry, P. M. Gruber and J. M. Wills (eds), vol B, North Holland, Amsterdam, 1993, 12971326.

[28] D. Hug, Generalized curvature measures and singularities of sets with positive reach, Forum Math. 10 (1998), 699-728.

[29] D. Hug, Absolute continuity for curvature measures of convex sets II, to appear in Math. Z.

[30] J.-B. Hiriart-Urruty and C. Lemaréchal, Convex Analysis and Minimization Algorithms I and II, Springer, Berlin, 1993.

[31] H. Ishii and P. L. Lions, Viscosity solutions of fully nonlinear second-order elliptic partial differential equations, J. Differential Equations 83 (1990), 26-78.

[32] R. Janin, Sur une classe de fonctions sous-linéarisables, C. R. Acad. Sci. Paris Sér. I Math. 277 (1973), 265-267.

[33] N. Kleinjohann, Nächste Punkte in der Riemannschen Geometrie, Math. Z. 176 (1981), 327-344.

[34] R. Rockafellar, Convex Analysis, Princeton University Press, Princeton, 1970.

[35] R. Rockafellar and R. Wets, Variational Analysis, Springer, Berlin, 1998.

[36] R. Schneider, Convex bodies: The Brunn-Minkowski theory, Cambridge University Press, Cambridge, 1993.

[37] R. Schneider, Convex surfaces, curvature and surface area measures, in Handbook of convex geometry, P. M. Gruber and J. M. Wills (eds), vol A, North Holland, Amsterdam, 1993, 273-299.

[38] R. Schneider, Simple valuations on convex bodies, Mathematika 43 (1996), 32-39.

[39] R. Schneider and J. A. Wieacker, Integral geometry, in Handbook of convex geometry, P. M. Gruber and J. M. Wills (eds), vol B, North Holland, Amsterdam, 1993, 13491390. 
[40] R. Schneider, Measures in convex geometry, Rend. Istit. Mat. Univ. Trieste 29 (1998), 215-265.

[41] N. Trudinger and X. J. Wang, Hessian measures I, Topological Methods in Nonlinear Analysis 10 (1997), 225-239.

[42] N. Trudinger and X. J. Wang, Hessian measures II, Preprint.

[43] F. A. Valentine, Convex Sets, McGraw-Hill, New York, 1964.

[44] X. Q. Yang, Generalised Hessian, Max function and weak convexity, Bull. Austral. Math. Soc. 53 (1996), 21-32.

Authors' addresses:

Andrea Colesanti

Dipartimento di Matematica "U. Dini"

Università degli Studi di Firenze

Viale Morgagni 67/A

50134 Firenze

Italy

andrea.colesanti@bb.math.unifi.it

Daniel Hug

Mathematisches Institut

Albert-Ludwigs-Universität

Eckerstraße 1

D-79104 Freiburg

Germany

hug@sun2.mathematik.uni-freiburg.de 